Michael Larsen · Alexander Lubotzky

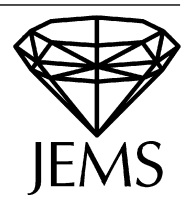

\title{
Representation growth of linear groups
}

Received April 22, 2007

\begin{abstract}
Let $\Gamma$ be a group and $r_{n}(\Gamma)$ the number of its $n$-dimensional irreducible complex representations. We define and study the associated representation zeta function $\mathcal{Z}_{\Gamma}(s)=$ $\sum_{n=1}^{\infty} r_{n}(\Gamma) n^{-s}$. When $\Gamma$ is an arithmetic group satisfying the congruence subgroup property then $\mathcal{Z}_{\Gamma}(s)$ has an "Euler factorization". The "factor at infinity" is sometimes called the "Witten zeta function" counting the rational representations of an algebraic group. For these we determine precisely the abscissa of convergence. The local factor at a finite place counts the finite representations of suitable open subgroups $U$ of the associated simple group $G$ over the associated local field $K$. Here we show a surprising dichotomy: if $G(K)$ is compact (i.e. $G$ anisotropic over $K$ ) the abscissa of convergence goes to 0 when $\operatorname{dim} G$ goes to infinity, but for isotropic groups it is bounded away from 0 . As a consequence, there is an unconditional positive lower bound for the abscissa for arbitrary finitely generated linear groups. We end with some observations and conjectures regarding the global abscissa.
\end{abstract}

\section{Introduction}

Let $\Gamma$ be a finitely generated group and let $s_{n}(\Gamma)$ denote the number of its subgroups of index at most $n$. The behavior of the sequence $\left\{s_{n}(\Gamma)\right\}_{n=1}^{\infty}$ and its relation to the algebraic structure of $\Gamma$ has been the focus of intensive research over the last two decades under the rubric "Subgroup Growth"- - see [LS] and the references therein.

Counting subgroups is essentially the same as counting permutation representations. In this paper we take a wider perspective: we count linear representations. So, let $r_{n}(\Gamma)$ be the number of $n$-dimensional irreducible complex representations of $\Gamma$. This number is not necessarily finite, in general (see $\$ 4$ below) but we consider only groups $\Gamma$ for which this is the case. In particular, it is so for the interesting family of irreducible lattices in higher-rank semisimple groups which will be our main cases of interest. By Margulis' arithmeticity theorem [Ma, p. 2], any such $\Gamma$ is commensurable to $\mathbf{G}\left(\mathcal{O}_{S}\right)$ where $\mathbf{G}$ is

This research was supported by grants from the NSF and the BSF (US-Israel Binational Science Foundation).

M. Larsen: Department of Mathematics, Indiana University, Bloomington, IN 47405, USA; e-mail: larsen@math.indiana.edu

A. Lubotzky: Institute of Mathematics, Hebrew University, Jerusalem 91904, Israel; e-mail: alexlub@math.huji.ac.il

Mathematics Subject Classification (2000): 11M41, 20E07, 22E35, 22E40 
an $\mathcal{O}_{S}$-subgroup scheme of $\mathrm{GL}_{d}$ with absolutely almost simple generic fiber. Here $k$ is a global field, $\mathcal{O}$ its ring of integers, $S$ a finite subset of $V$, the set of valuations of $k$, containing $V_{\infty}$, the set of archimedean valuations, and $\mathcal{O}_{S}$ the ring of $S$-integers.

The (finite-dimensional complex) representation theory of $\Gamma$ is captured by the group $A(\Gamma)$, the proalgebraic completion of $\Gamma$. In $\S 2$, we present some background and basic results on $A(\Gamma)$. If $\Gamma=\mathbf{G}\left(\mathcal{O}_{S}\right)$ as before and if in addition $\Gamma$ has the congruence subgroup property (CSP, for short), i.e.

$$
C(\Gamma):=\operatorname{ker}\left(\widehat{\mathbf{G}\left(\mathcal{O}_{S}\right)} \rightarrow \mathbf{G}\left(\hat{\mathcal{O}}_{S}\right)\right)
$$

is finite, then $A(\Gamma)$ can be described quite precisely:

Proposition 1.1. Let $\Gamma=\mathbf{G}\left(\mathcal{O}_{S}\right)$ as before and assume $\Gamma$ has the congruence subgroup property. Then $A(\Gamma)$ has a finite normal subgroup $C$ isomorphic to $C(\Gamma)=$ $\operatorname{ker}\left(\widehat{\mathbf{G}\left(\mathcal{O}_{S}\right)} \rightarrow \mathbf{G}\left(\hat{\mathcal{O}}_{S}\right)\right)$ such that

$$
A(\Gamma) / C \cong \mathbf{G}(\mathbb{C})^{r} \times \prod_{v \in V_{f} \backslash S} \mathbf{G}\left(\mathcal{O}_{v}\right)
$$

where $r$ is the number of archimedean valuations of $k, V_{f}=V \backslash V_{\infty}$, and $\mathcal{O}_{v}$ is the completion of $\mathcal{O}$ with respect to a finite valuation $v$.

Note that $A(\Gamma)$ is a direct product of its identity component $\mathbf{G}(\mathbb{C})^{r}$ and $\hat{\Gamma}$, the profinite completion of $\Gamma$. Moreover, $\Gamma$ is embedded in $\mathbf{G}(\mathbb{C})^{r}$ via the diagonal map: $\Gamma=$ $\mathbf{G}\left(\mathcal{O}_{S}\right) \rightarrow \prod_{v \in V_{\infty}} \mathbf{G}\left(k_{v}\right) \leq \mathbf{G}(\mathbb{C})^{r}$.

Implicit in the proposition is the fact that the CSP implies super-rigidity: If $\rho$ is a finite-dimensional complex representation of $\Gamma$ then it can be extended on some finite index subgroup to a rational representation of $\mathbf{G}(\mathbb{C})^{r}$.

Recall now that Serre's conjecture $[\mathrm{Se}]$ asserts that if $\mathbf{G}$ is simply connected and $\sum_{v \in S} \mathrm{rk}_{k_{v}}(\mathbf{G}) \geq 2$ then $\Gamma$ has the CSP. In most cases this has been proved (see [PR] $\S 9.5]$ and the references therein). Moreover, in [LuMr] it is shown that if $\Gamma$ has the CSP then $r_{n}(\Gamma)$ is polynomially bounded when $n \rightarrow \infty$. (It is further shown that if $\operatorname{char}(k)=0$ this property is equivalent to the CSP and it is conjectured that the same is true in general). Let us now define:

Definition 1.2. The representation zeta function of $\Gamma$ is defined to be

$$
\mathcal{Z}_{\Gamma}(s)=\sum_{n=1}^{\infty} r_{n}(\Gamma) n^{-s} .
$$

Its abscissa of convergence is

$$
\rho(\Gamma)=\limsup _{n \rightarrow \infty} \frac{\log R_{n}(\Gamma)}{\log n}
$$

where $R_{n}(\Gamma)=\sum_{i=1}^{n} r_{i}(\Gamma)$, the number of irreducible representations of degree at most $n$. 
Our main goal in this paper is to initiate the study of representation zeta functions of arithmetic groups $\Gamma$, in analogy with the theory of subgroup zeta functions of nilpotent groups (cf. [DG] and [LS, Chapters 15 and 16]).

So, if $\Gamma$ has the CSP then $\rho(\Gamma)<\infty$. The study of $\rho(\Gamma)$ will be one of our main goals. This makes sense for any finitely generated group. If $R_{n}(\Gamma)$ is not polynomially bounded (in particular, if $R_{n}(\Gamma)$ is infinite for some $n$ ) we simply write $\rho(\Gamma)=\infty$.

Assume for simplicity now that $\Gamma$ has the CSP and the congruence kernel $C(\Gamma)$ is trivial. Proposition 1.1 then implies the important "Euler factorization" of $\mathcal{Z}_{\Gamma}(s)$.

Proposition 1.3. If $\Gamma=\mathbf{G}\left(\mathcal{O}_{S}\right)$, $\Gamma$ has the $\operatorname{CSP}$ and $C(\Gamma)=\{e\}$ then

$$
\mathcal{Z}_{\Gamma}(s)=\mathcal{Z}_{\mathbf{G}(\mathbb{C})}(s)^{r} \times \prod_{v \in V_{f} \backslash S} \mathcal{Z}_{\mathbf{G}\left(\mathcal{O}_{v}\right)}(s) .
$$

Of course, here we are using the notation $\mathcal{Z}_{H}(s)$ for groups $H$ which are not discrete. When $H$ is a profinite group (resp. the group of real or complex points of an algebraic group), we count only continuous (resp. rational) representations.

A concrete example to think about is $\Gamma=\mathrm{SL}_{3}(\mathbb{Z})$ for which

$$
\mathcal{Z}_{\mathrm{SL}_{3}(\mathbb{Z})}(s)=\mathcal{Z}_{\mathrm{SL}_{3}(\mathbb{C})}(s) \times \prod_{p} \mathcal{Z}_{\mathrm{SL}_{3}\left(\mathbb{Z}_{p}\right)}(s)
$$

So, we have an Euler factorization with $p$-adic factors as well as a factor at infinity. We note here that the $p$ th local factor is not quite a power series in $p^{-s}$, i.e., it does not count the irreducible representations of $p$-power degrees, but this is not too far from the truth as $\mathrm{SL}_{3}\left(\mathbb{Z}_{p}\right)$ is a virtually pro- $p$ group (see $\S 4$ and $\S 6$ ). Anyway, we can define $\rho_{\infty}(\Gamma)$ to be the abscissa of convergence of the identity component of $A(\Gamma)$, i.e. of $\mathbf{G}(\mathbb{C})^{r}$. But as $\mathcal{Z}_{\mathbf{G}(\mathbb{C})^{r}}(s)=\left(\mathcal{Z}_{\mathbf{G}(\mathbb{C})}(s)\right)^{r}$ this is equal to $\rho(\mathbf{G}(\mathbb{C}))$. The factor of infinite $\mathcal{Z}_{\mathbf{G}(\mathbb{C})}(s)$, the so-called "Witten zeta function", is discussed in $\$ 5$ below.

Similarly for every $v \in V_{f}$ we have $\rho_{v}(\Gamma)=\rho\left(\mathbf{G}\left(\mathcal{O}_{v}\right)\right)$, the $v$-local abscissa of convergence.

Theorem 5.1. For $\mathbf{G}$ as before,

$$
\rho(\mathbf{G}(\mathbb{C}))=r / \kappa
$$

where $r=\operatorname{rk} \mathbf{G}=\left(\right.$ absolute) rank of $\mathbf{G}$ and $\kappa=\left|\Phi^{+}\right|$where $\Phi^{+}$is the set of positive roots in the absolute root system associated to $\mathbf{G}$.

Note that $\kappa=\left|\Phi^{+}\right|=\frac{1}{2}(\operatorname{dim} \mathbf{G}-\operatorname{rk} \mathbf{G})$ and $r / \kappa=2 / h$ where $h$ is the Coxeter number of $\Phi$.

The expression $r / \kappa$ has already appeared in an analogous context in the work of Liebeck and Shalev:

Theorem 1.4 (Liebeck-Shalev [LiSh2]). Let $\mathbf{G}$ be a Chevalley group scheme over $\mathbb{Z}$. Then

$$
\limsup _{n, q \rightarrow \infty} \frac{\log R_{n}\left(\mathbf{G}\left(\mathbb{F}_{q}\right)\right)}{\log n}=\frac{r}{\kappa} \text {. }
$$


For $\mathbf{G}\left(\mathcal{O}_{v}\right)$ as above, we prove:

Proposition $6.6 \rho\left(\mathbf{G}\left(\mathcal{O}_{v}\right)\right) \geq r / \kappa$.

In the anisotropic case in characteristic zero, we can prove equality:

Theorem7.1 If $\mathbf{G}(K)=\mathrm{SL}_{1}(D)$ where $D$ is a division algebra of degree d over a local field $K$ of characteristic 0 , then $\mathbf{G}(K)$ is a compact virtually pro- $p$ group and

$$
\rho(\mathbf{G}(K))=\frac{r}{\kappa}=\frac{2}{d} .
$$

Jaikin-Zapirain [Ja2] computed the $v$-adic local zeta function of $\operatorname{SL}_{2}\left(\mathcal{O}_{v}\right)$. From his result one sees that $\rho=1=r / \kappa$ for all such groups.

All these examples suggested to us that $\rho\left(\mathbf{G}\left(\mathcal{O}_{v}\right)\right)$ would always be equal to $r / \kappa$. The truth, however, is quite different:

Theorem 8.1 If $K$ is a non-archimedean local field, $\mathbf{G}$ an isotropic simple $K$-group, and $U$ an open compact subgroup of $\mathbf{G}(K)$, then $\rho(U) \geq \frac{1}{15}$.

We remark that $1 / 15$ is probably not the best possible constant. It is dictated by the fact that for $E_{8}$ (and for other exceptional groups with smaller Coxeter number), we do not know how to improve on the bound of Proposition 6.6 We also note that for nonarchimedean local fields $K$, the only anisotropic groups are those of the type $\mathbf{G}(K)=$ $\mathrm{SL}_{1}(D)$ described in Theorem 7.1. For these, $r / \kappa$ goes to zero when $\operatorname{dim} D$ goes to infinity. So Theorems 8.1 and 7.1 give a dichotomy between isotropic and anisotropic groups. The latter case we understand well: we can estimate the number of representations of given degree by counting coadjoint orbits. In the former case, there is a distinction between $\mathbf{G}(K)$-orbits and $\mathbf{G}\left(\mathcal{O}_{v}\right)$-orbits, which appears to be controlled by the rate of growth of balls in the Bruhat-Tits building of $\mathbf{G}$ over $K$. When this rate of growth is high enough, it dominates the estimates of representation growth. Unfortunately, we still do not know how to compute the precise rates of growth in this case. (See $\$ 11$ below for more on this point of view, which suggested the computations of $\S 8$ but is not made explicit there.)

An unexpected consequence of Theorem 8.1 is

Theorem 9.1 If $\Gamma$ is a finitely generated group with some linear representation $\varphi: \Gamma \rightarrow$ $\mathrm{GL}_{n}(F)$, with $F$ a field, such that $\varphi(\Gamma)$ is infinite (e.g. $\Gamma$ an infinite linear group) then $\rho(\Gamma) \geq 1 / 15$.

On the other hand, we show in $\S 9$ that there exist infinite, finitely generated, residually finite groups $\Gamma$ with $\rho(\Gamma)=0$.

In $\S 10$, we analyze $\rho(\Gamma)$ for arithmetic lattices in semisimple groups of a very special type, namely, powers of $\mathrm{SL}_{2}$. These are very special cases (and, as we saw above, in this problem special cases can be quite misleading.) We still believe in the conjecture these examples suggest:

Conjecture 1.5. Let $H$ be a higher rank semisimple group (i.e. $H$ is a product $\prod_{i=1}^{\ell} G_{i}\left(K_{i}\right)$ where each $K_{i}$ is a local field, each $G_{i}$ is an absolutely almost simple $K_{i}$-group, and $\sum_{i=1}^{\ell} \operatorname{rk}_{K_{i}}\left(G_{i}\right) \geq 2$ ). Then for any two irreducible lattices $\Gamma_{1}$ and $\Gamma_{2}$ in $H, \rho\left(\Gamma_{1}\right)=\rho\left(\Gamma_{2}\right)$. 
This last conjecture should be compared with [LuNi, Theorem 11] concerning the growth of $s_{n}(\Gamma)$, the number of subgroups of index less than or equal to $n$, in an irreducible lattice of a higher rank semisimple group:

Theorem 1.6 (Lubotzky-Nikolov [LuNi]). Let $H$ be a higher rank semisimple group. Assuming the GRH (generalized Riemann hypothesis) and Serre's conjecture, for every irreducible lattice $\Gamma$ in $H$, the limit

$$
\lim _{n \rightarrow \infty} \frac{\log s_{n}(\Gamma)}{(\log n)^{2} / \log \log n}
$$

exists and equals $\tau(H)$, an invariant of $H$ which is given explicitly in [LuNi].

See [LuNi] for further information, including many cases for which the theorem is proved unconditionally.

Theorem 1.6 says that the subgroup growth (i.e., the permutation representation rate of growth) is very similar for different irreducible lattices in $H$. Conjecture 1.5 makes a similar statement regarding their finite-dimensional complex representations.

There is still a significant difference. While in [LuNi] a precise formula is given for $\tau(H)$, so far we do not even have a guess what will be the common value predicted by Conjecture 1.5. It seems likely that one needs first to understand the local abscissas of convergence, but even knowing them in full does not necessarily give the global abscissa.

The paper is organized as follows: in $\S 2$ we describe $A(\Gamma)$, the proalgebraic completion, and $B(\Gamma)$, the Bohr compactification, of a higher rank arithmetic group $\Gamma$. In $\S 3$ and $\$ 4$ we show how the congruence subgroup property gives the precise structure of $A(\Gamma)$ and out of this an Euler factorization is deduced for $\mathcal{Z}_{\Gamma}(s)$. The factor at infinity is studied in $\$ 5$ where a precise formula is given for its abscissa of convergence (Theorem 5.1). The finite local factors are studied in $\$ 6$ (generalities), §7 (the anisotropic case-Theorem 7.1), and in $\$ 8$ (the isotropic case-Theorem 8.1). The applications to discrete groups are derived in $\S 9$. In $\S 10$, we give some evidence for Conjecture 1.5 . We end in $\$ 11$ with remarks and suggestions for further research. It seems that our results reveal only the tip of the iceberg of $\mathcal{Z}_{\Gamma}(s)$.

Conventions. In this paper representations always mean complex finite-dimensional representations.

We study representation theory of various discrete groups $\Gamma$ which are always assumed to be finitely generated.

\section{The proalgebraic completion and Bohr compactification of arithmetic groups}

Let $\Gamma$ be a finitely generated group. A useful tool for studying the finite-dimensional representation theory of $\Gamma$ over $\mathbb{C}$ is the proalgebraic completion $A(\Gamma)$ of $\Gamma$, known also as the Hochschild-Mostow group of $\Gamma$. (See $[\overline{\mathrm{HM}}],[\mathrm{LuMg}]$ and $[\mathrm{BLMM}]$ for a systematic description.) The group $A(\Gamma)$ together with the structure homomorphism

$$
i: \Gamma \rightarrow A(\Gamma)
$$


is uniquely characterized by the following property: For every representation $\rho$ of $\Gamma$ there is a unique rational representation $\bar{\rho}$ of $A(\Gamma)$ such that $\bar{\rho} \circ i=\rho$.

This implies that the representation theory of $\Gamma$ is equivalent to the rational representation theory of $A(\Gamma)$. The image $\bar{\rho}(A(\Gamma))$ is always the Zariski closure of $\rho(\Gamma)$ and in fact, $A(\Gamma)$ is the inverse limit of these closures over all representations of $\Gamma$. In particular, $A(\Gamma)$ is mapped onto the profinite completion $\hat{\Gamma}$ of $\Gamma$ (which can be thought as the inverse limit over the representations with finite image). The kernel $A(\Gamma)^{\circ}$ of the exact sequence

$$
1 \rightarrow A(\Gamma)^{\circ} \rightarrow A(\Gamma) \rightarrow \hat{\Gamma} \rightarrow 1
$$

is the connected component of $A(\Gamma)$. It is a simply connected proaffine algebraic group [BLMM, Theorem 1]

The group $\Gamma$ is called super-rigid if $A(\Gamma)$ is finite-dimensional (i.e., $A(\Gamma)^{\circ}$ is finitedimensional). It is shown in [BLMM, Theorem 5] that if $\Gamma$ is linear over $\mathbb{C}$ and super-rigid then it has a finite index normal subgroup $\Gamma_{0}$ such that $A\left(\Gamma_{0}\right) \simeq A\left(\Gamma_{0}\right)^{\circ} \times \hat{\Gamma}_{0}$.

It can be easily seen that $\Gamma_{0}$ can be chosen so that $\Gamma_{0} \rightarrow A\left(\Gamma_{0}\right)^{\circ}$ is injective and every representation of $\Gamma$ can be extended, on a finite index subgroup $\Gamma_{1}$ of $\Gamma_{0}$ (and therefore of $\Gamma$ ), to a rational representation of $A\left(\Gamma_{0}\right)^{\circ}=A(\Gamma)^{\circ}$. (Note that for a finite-dimensional rational representation of $A\left(\Gamma_{0}\right)$, the image of $\hat{\Gamma}_{0}$ is finite). So, super-rigidity for a linear group $\Gamma$ implies, and in fact is equivalent to, the existence of a finite-dimensional connected, simply connected, algebraic group $G$ containing a finite index subgroup $\Gamma_{0}$ of $\Gamma$, such that every representation of $\Gamma$ can be extended to $G$ on some finite index subgroup of $\Gamma_{0}$.

As is well known, Margulis' super-rigidity theorem [Ma, p. 2] says that irreducible lattices $\Gamma$ in higher rank semisimple groups $H$ are super-rigid. (This has now been supplemented ([Co], [GS]) for lattices in $\operatorname{Sp}(n, 1), n \geq 1$, and $F_{4}^{(-20)}$.) Margulis' arithmeticity theorem [Ma, p. 2] (which is deduced from the super-rigidity) says that every such $\Gamma$ is $(S$-) arithmetic.

Let us now spell out the precise meaning of this regarding $A(\Gamma)$. So let $H$ be a semisimple (locally compact) group. By this we mean

$$
H=\prod_{i=1}^{\ell} G_{i}\left(K_{i}\right)
$$

where each $K_{i}$ is a local field and $G_{i}$ is an absolutely almost simple group defined over $K_{i}$. We assume that no $G_{i}\left(K_{i}\right)$ is compact, i.e., $\operatorname{rk}_{K_{i}}\left(G_{i}\right) \geq 1$.

If $\sum_{i=1}^{\ell} \mathrm{rk}_{K_{i}}\left(G_{i}\right) \geq 2$; or if $\ell=1, K_{1}=\mathbb{R}$, and $G_{1}(\mathbb{R})$ is locally isomorphic to one of the real rank one groups $\operatorname{Sp}(n, 1)$ or $F_{4}^{(-20)}$, then every irreducible lattice of $H$ is arithmetic. This means that there exists a global field $k$, a finite set $S$ of valuations of $k$ containing all the archimedean ones, with $\mathcal{O}_{S}=\{x \in k \mid v(x) \geq 0 \forall v \notin S\}$, and a group scheme $\mathbf{G} / \mathcal{O}_{S}$ of finite type whose generic fiber is connected, simply connected and semisimple, with a continuous map $\psi: \prod_{v \in S} \mathbf{G}\left(k_{v}\right) \rightarrow H$ whose kernel and cokernel are compact and such that $\psi\left(\mathbf{G}\left(\mathcal{O}_{S}\right)\right)$ is commensurable to $\Gamma$. (We note that the scheme can be chosen to be flat—see [BLR, 1.1].) 
This in particular implies that if an irreducible lattice in $H$ exists, then all the fields $K_{i}$ are of the same characteristic, and all the algebraic groups $G_{i}$ are forms of the same group. It also says that such a lattice $\Gamma$ is isomorphic, up to finite index, to $\mathbf{G}\left(\mathcal{O}_{S}\right)$.

We can now describe the pro-algebraic completion of $\mathbf{G}\left(\mathcal{O}_{S}\right)$ :

Theorem 2.1. With the notation of $\mathbf{G}\left(\mathcal{O}_{S}\right)$ as above (including the assumption $\sum_{\text {have }} \mathrm{rk}_{k_{v}}(\mathbf{G}) \geq 2$; or $\ell=1, K_{1}=\mathbb{R}$, and $G_{1}\left(K_{1}\right)$ is either $\operatorname{Sp}(n, 1)$ or $F_{4}^{(-20)}$ ) we

$$
A\left(\mathbf{G}\left(\mathcal{O}_{S}\right)\right)=\mathbf{G}(\mathbb{C})^{\# S_{\infty}} \times \widehat{\mathbf{G}\left(\mathcal{O}_{S}\right)}
$$

where $S_{\infty}$ is the set of archimedean valuations of $k$.

Proof. If $k$ is of positive characteristic then by [Ma, Theorem 3, p. 3], $A\left(\mathbf{G}\left(\mathcal{O}_{S}\right)\right)=$ $\widehat{\mathbf{G}\left(\mathcal{O}_{S}\right)}$ and we are done. Assume $\operatorname{char}(k)=0$ and then by the same theorem, for every complex representation of $\Gamma=\mathbf{G}\left(\mathcal{O}_{S}\right)$, the identity component $\bar{\Gamma}^{\circ}$ of the Zariski closure of $\Gamma$ is semisimple. By [Ma, Theorem 5, p. 5] every such representation of $\Gamma$, or of a finite index subgroup thereof, into a simple algebraic $\mathbb{C}$-group is obtained (up to finite index subgroup) by embedding $\mathcal{O}_{S}$ into $\mathbb{C}$ and then composing with an algebraic representation of $\mathbf{G}(\mathbb{C})$.

We can therefore deduce that with $\Gamma$ embedded diagonally in $M=\prod_{v \in S_{\infty}} \mathbf{G}(\mathbb{C})$, every complex representation of $\Gamma$ can be extended, on a finite index subgroup of $\Gamma$, to a representation of $M$. This proves that $A(\Gamma)^{\circ} \cong M$.

We have a direct product decomposition $A(\Gamma)=A(\Gamma)^{\circ} \times \hat{\Gamma}$ since $\Gamma$ is indeed densely embedded in $M=A(\Gamma)^{\circ}$ and hence there is a map $A(\Gamma) \rightarrow A(\Gamma)^{\circ}$.

So super-rigidity gives the complete description of $A(\Gamma)^{\circ}$. We should now concentrate on $\hat{\Gamma}=\widehat{\mathbf{G}\left(\mathcal{O}_{S}\right)}$. Here we need the congruence subgroup property to be discussed in the next section. We mention here in passing that super-rigidity also gives the complete description of the Bohr compactification of $\Gamma$. Let us first recall:

Definition 2.2. For a finitely generated group $\Gamma$ we denote by $B(\Gamma)$ its Bohr compactification. This is a compact group together with a homomorphism $j: \Gamma \rightarrow B(\Gamma)$ with the following universal property: If $\varphi$ is a homomorphism of $\Gamma$ into some compact group $K$, there exists a unique continuous extension $\tilde{\varphi}: B(\Gamma) \rightarrow K$ with $\tilde{\varphi} \circ j=\varphi$.

The existence of such $B(\Gamma)$ (and $j$ ) is easy to establish: Let $\left\{C_{\alpha}, \psi_{\alpha}\right\}$ be the family of all possible homomorphisms $\psi_{\alpha}: \Gamma \rightarrow K_{\alpha}$ where $K_{\alpha}$ is a compact group. Take $C=$ $\prod_{\alpha} K_{\alpha}$. Then $B(\Gamma)$ is the closure of the image of $\Gamma$ in $C$ under the diagonal map $\gamma \mapsto$ $\left(\psi_{\alpha}(\gamma)\right)_{\alpha}$ for $\gamma \in \Gamma$. The Bohr compactification is of importance in the theory of almost periodic functions ([Cd, Chapter VII]).

Proposition 2.3. Let $\Gamma=\mathbf{G}\left(\mathcal{O}_{S}\right)$ be as in Theorem 2.1. Then

$$
B(\Gamma)=\prod_{\sigma \in T}{ }^{\sigma} \mathbf{G}(\mathbb{R}) \times \widehat{\mathbf{G}\left(\mathcal{O}_{S}\right)}
$$

where $T$ is the set of all real embeddings of $k$ for which ${ }^{\sigma} \mathbf{G}(\mathbb{R})$ is compact, where ${ }^{\sigma} \mathbf{G}=$ $\mathbf{G} \times{ }_{\sigma} \mathbb{R}$. Note that $T$ can be considered as a subset of $S_{\infty}$. 
Proof. By the Peter-Weyl theorem every compact group is an inverse limit of finitedimensional compact Lie groups. Let $L=\prod_{\sigma \in T}{ }^{\sigma} \mathbf{G}(\mathbb{R})$. To prove that $B(\Gamma)^{\circ}=L$ means proving that if $\psi: \Gamma \rightarrow K$ is a homomorphism of $\Gamma$ into a dense subgroup of a compact Lie group $K$, then $\psi$ can be extended, up to a finite index subgroup, to a continuous homomorphism from $L$ to $K$.

As $K$ is compact, its identity component is the group of real points of a real connected algebraic group, $K^{\circ}=H(\mathbb{R})$. Again, as in the proof of Theorem 2.1, if $\operatorname{char}(k)>0$, then $\psi$ has finite image and $B(\Gamma)=\hat{\Gamma}$. If $\operatorname{char}(k)=0, H$ is semisimple and each one of its almost simple factors is absolutely almost simple over $\mathbb{R}$ (otherwise, it would be a restriction of scalars of a complex group and hence not compact). We can use $\overline{\mathrm{Ma}}$, Theorem 5, p. 5] again to deduce that the connected component of $B(\Gamma)$ is indeed $L$. As before, it is a direct factor since we have a dense map from $\Gamma$ to $L$.

\section{The congruence subgroup property}

We continue with the notation of the previous section. So $\mathbf{G}$ is a group scheme of finite type over $\mathcal{O}_{S}$, the ring of $S$-integers in a global field $k$, whose generic fiber is connected, simply connected, and absolutely almost simple, and $\Gamma=\mathbf{G}\left(\mathcal{O}_{S}\right)$.

Definition 3.1. The group $\Gamma$ is said to have the congruence subgroup property (CSP for short) if $\operatorname{ker}\left(\widehat{\mathbf{G}\left(\mathcal{O}_{S}\right)} \stackrel{\pi}{\rightarrow} \mathbf{G}\left(\hat{\mathcal{O}}_{S}\right)\right)$ is finite.

Now by the strong approximation theorem (cf. [PR, Theorem 7.12] and $[\mathrm{Pr}]) \pi$ is onto. Moreover, $\mathbf{G}\left(\hat{\mathcal{O}}_{S}\right)=\prod_{v \notin S} \mathbf{G}\left(\mathcal{O}_{v}\right)$. Note that if $\Gamma$ has the CSP then by replacing $\Gamma$ with a suitable finite index subgroup $\Gamma_{0}$, we have $\hat{\Gamma}_{0}=\prod_{v \notin S} L_{v}$, where $L_{v}$ is open in $\mathbf{G}\left(\mathcal{O}_{v}\right)$ for every $v$ and equal to it for almost every $v$.

Before continuing, let us recall (see [BMS, §16], [Se, §2.7], and [Ra, Theorem 7.2]) that the CSP implies super-rigidity. In our language this means

Theorem 3.2. If $\Gamma=\mathbf{G}\left(\mathcal{O}_{S}\right)$ has the CSP then $A(\Gamma)^{\circ}$ is finite-dimensional.

Sketch of proof. First consider a representation $\rho: \Gamma \rightarrow \mathrm{GL}_{n}(\mathbb{Q})$. Unless $\Gamma$ is a lattice in a rank one group over a positive characteristic field, in which case $\Gamma$ does not have the CSP (see [Lu2, Theorem D]), $\Gamma$ is finitely generated and hence the entries of $\rho(\Gamma)$ are $p$-adic integers for almost every prime $p$. Choose such a prime $p$ (which is not $\operatorname{char}(k)$ ). Thus we have a representation into $\mathrm{GL}_{n}\left(\mathbb{Z}_{p}\right)$. This last group has a finite index torsionfree pro- $p$ subgroup $H$. Now, if $\Gamma$ has CSP, then after passing to a finite index subgroup $\Gamma_{0}$ of $\Gamma, \hat{\Gamma}_{0}=\prod_{v \notin S} L_{v}$ where $L_{v}$ is open in $\mathbf{G}\left(\mathcal{O}_{v}\right)$. If $\operatorname{char}(k)=\ell>0$ then $L_{v}$ is a virtually pro- $\ell$ group and so its image in $H$ is finite and hence trivial. This proves that $\rho(\Gamma)$ was finite to start with. If $\operatorname{char}(k)=0$ then for every $v$ which does not lie over $p$, $\rho\left(L_{v}\right)$ is finite and again trivial. So we get a map from $\prod_{v \mid p} L_{v}$ to $\mathrm{GL}_{n}\left(\mathbb{Z}_{p}\right)$. This is a map between two $p$-adic analytic virtually pro- $p$ groups, which must be analytic and in fact algebraic as $\mathbf{G}$ is semisimple. Thus altogether, $\rho$ can be extended, on a finite index subgroup, to an algebraic representation of $\mathbf{G}$. 
The above proof works word for word also for representations over number fields and hence also with regard to representations into $\mathrm{GL}_{n}(\overline{\mathbb{Q}})$, where $\overline{\mathbb{Q}}$ is an algebraic closure of $\mathbb{Q}$. This implies in particular that $\Gamma$ has only finitely many irreducible $n$-dimensional $\overline{\mathbb{Q}}$-representations. Indeed, if $\Gamma$ has the CSP then it has FAb, i.e., $|\Delta /[\Delta, \Delta]|<\infty$ for every finite index subgroup $\Delta$ of $\Gamma$. It follows now from Jordan's theorem (cf. [LS, p. 376]; see also [BLMM, Cor. 8]) that $\Gamma$ has only finitely many $n$-dimensional representations with finite image. The same also applies to algebraic representations of $\mathbf{G}$. By the Nullstellensatz the same applies to representations over $\mathbb{C}$. So the character variety is finite $($ see $[\mathrm{LuMg}])$ and all the representations can be conjugated into $\mathrm{GL}_{n}(\overline{\mathbb{Q}})$.

Note also that if $\Gamma$ has the CSP then by replacing $\Gamma$ by a suitable finite index $\Gamma_{0}$ as before, $\hat{\Gamma}_{0}=\prod_{v} L_{v}$, and combining this with the proof of Theorem 2.1 above we get:

Theorem 3.3. If $\Gamma=\mathbf{G}\left(\mathcal{O}_{S}\right)$ has the CSP then for a suitable finite index subgroup $\Gamma_{0}$ of $\Gamma\left(\right.$ with $\Gamma_{0}=\Gamma$ if $\left.\operatorname{ker}\left(\widehat{\mathbf{G}\left(\mathcal{O}_{S}\right)} \rightarrow \mathbf{G}\left(\hat{\mathcal{O}}_{S}\right)\right)=\{e\}\right)$,

$$
A\left(\Gamma_{0}\right)=\mathbf{G}(\mathbb{C})^{\# S_{\infty}} \times \prod_{v \notin S} L_{v}
$$

where $L_{v}$ is open in $\mathbf{G}\left(\mathcal{O}_{v}\right)$ and equal to it for almost all $v$.

Finally, we mention the main result of $[\overline{\mathrm{LuMr}}]$ :

Theorem 3.4 (Lubotzky-Martin [ $\overline{\mathrm{LuMr}}])$. If $\Gamma=\mathbf{G}\left(\mathcal{O}_{S}\right)$ has the CSP then $r_{n}(\Gamma)$ is polynomially bounded. If $\operatorname{char}(k)=0$ then the converse is also true.

It is conjectured in [LuMr] that the converse also holds if $\operatorname{char}(k)>0$, and some steps in this direction are taken there.

\section{The representation zeta function}

Let $\Gamma$ be a finitely generated group and $r_{n}(\Gamma)$ the number of its $n$-dimensional irreducible complex representations. This may not be a finite number. Similarly, denote by $\hat{r}_{n}(\Gamma)$ the number of $n$-dimensional irreducible representations of $\Gamma$ with finite image.

Proposition 4.1 ([BLMM, Proposition 2]). We have $\hat{r}_{n}(\Gamma)<\infty$ for every $n$ if and only if $\Gamma$ has $(\mathrm{FAb})$, i.e. $|\Delta /[\Delta, \Delta]|<\infty$ for every finite index subgroup $\Delta$ of $\Gamma$.

On the other hand, there is no known intrinsic characterization of groups $\Gamma$ for which $r_{n}(\Gamma)<\infty$ for every $n$. Such a group is called rigid.

Problem 4.2. Characterize rigid groups.

Anyway, we assume from now on that $\Gamma$ is rigid and define: 
Definition 4.3. (a) The representation zeta function of $\Gamma$ is

$$
\mathcal{Z}_{\Gamma}(s)=\sum_{n=1}^{\infty} r_{n}(\Gamma) n^{-s},
$$

and the finite representation zeta function is

$$
\hat{\mathcal{Z}}_{\Gamma}(s)=\sum_{n=1}^{\infty} \hat{r}_{n}(\Gamma) n^{-s} .
$$

(b) Let $\rho(\Gamma)=\varlimsup \frac{\log R_{n}(\Gamma)}{\log n}$ where $R_{n}(\Gamma)=\sum_{i=1}^{n} r_{i}(\Gamma)$. It is called the abscissa of convergence of $\mathcal{Z}_{\Gamma}(s)$.

The following easy result is given in [LuMr, Lemma 2.2]:

Proposition 4.4. If $\Gamma_{0}$ is a subgroup of index $m$ in $\Gamma$ then

$$
R_{n}\left(\Gamma_{0}\right) \leq m R_{m n}(\Gamma) \quad \text { and } \quad R_{n}(\Gamma) \leq m R_{n}\left(\Gamma_{0}\right) .
$$

Corollary 4.5. $\rho\left(\Gamma_{0}\right)=\rho(\Gamma)$.

Now, if $\rho(\Gamma)<\infty$ then $\mathcal{Z}_{\Gamma}(s)$ indeed defines a holomorphic function on the half-plane $\{s \in \mathbb{C} \mid \operatorname{Re} s>\rho(\Gamma)\}$ and $r_{n}(\Gamma)$ is polynomially bounded.

Let now $\Gamma=\mathbf{G}\left(\mathcal{O}_{S}\right)$ as in Section 3. Assume further that $\Gamma$ has the CSP. Then by Theorem 3.4 $\rho(\Gamma)<\infty$ and $\mathcal{Z}_{\Gamma}(s)$ is indeed a well defined function on the halfplane. Moreover, let $\Gamma_{0}$ be a finite index subgroup of $\Gamma$, as in $\S 3$, for which $A\left(\Gamma_{0}\right)=$ $\mathbf{G}(\mathbb{C})^{\# S_{\infty}} \times \prod_{v \notin S} L_{v}$ with $L_{v}$ open in $\mathbf{G}\left(\mathcal{O}_{v}\right)$ for every $v$ and $L_{v}=\mathbf{G}\left(\mathcal{O}_{v}\right)$ for almost every $v$. (We can take $\Gamma_{0}=\Gamma$ if $\operatorname{ker}\left(\widehat{\mathbf{G}\left(\mathcal{O}_{S}\right)} \rightarrow \mathbf{G}\left(\hat{\mathcal{O}}_{S}\right)\right)=\{e\}$.) Since there is a oneto-one correspondence between representations of $\Gamma$ and rational representations of $A(\Gamma)$ and since every irreducible representation of a product of groups decomposes in a unique way as a tensor product of irreducible representations of the factor groups, we get an "Euler factorization":

\section{Proposition 4.6.}

$$
\mathcal{Z}_{\Gamma_{0}}(s)=\mathcal{Z}_{\mathbf{G}(\mathbb{C})}(s)^{\# S_{\infty}} \cdot \prod_{v \notin S} \mathcal{Z}_{L_{v}}(s)
$$

where $\mathcal{Z}_{\mathbf{G}(\mathbb{C})}(s)$ (resp. $\mathcal{Z}_{L_{v}}(s)$ ) is the representation zeta function counting the irreducible rational (resp. continuous) representations of $\mathbf{G}(\mathbb{C})$ (resp. $\left.L_{v}\right)$.

Now if we look at $V(p)=\{v|v \notin S, v| p\}$, i.e. all the valuations of $k$ (outside $S$ ) which lie over a prime $p$, then $\prod_{v \in V(p)} \mathcal{Z}_{L_{v}}(s)$ will be called the $p$-factor of $\mathcal{Z}_{\Gamma}(s)$ and it will be denoted $\mathcal{Z}_{\Gamma}^{p}(s)$. Similarly, $\mathcal{Z}_{\mathbf{G}(\mathbb{C})}(s)^{\# S_{\infty}}$ is the infinite (or archimedean) factor of the "Euler factorization".

It should be noted that unlike the classical Euler factorization, $\mathcal{Z}_{\Gamma}^{p}(s)$ does not exactly encode the representations of $p$-power dimension. 
Example 4.7. Let $\Gamma=\mathrm{SL}_{3}(\mathbb{Z})$, so

$$
A(\Gamma)=\mathrm{SL}_{3}(\mathbb{C}) \times \prod_{p} \mathrm{SL}_{3}\left(\mathbb{Z}_{p}\right)
$$

and $\mathcal{Z}_{\Gamma}(s)=\mathcal{Z}_{\mathrm{SL}_{3}(\mathbb{C})} \times \prod_{p} \mathcal{Z}_{\mathrm{SL}_{3}\left(\mathbb{Z}_{p}\right)}(s)$. The degrees of the irreducible representations of the pro-finite group $\mathrm{SL}_{3}\left(\mathbb{Z}_{p}\right)$ divide its order (which is a super-natural number-see [Ri, §1.4]). As $\mathrm{SL}_{3}\left(\mathbb{Z}_{p}\right)$ is a virtually pro- $p$ group the set of these degrees is contained in a finite union of type $\bigcup_{j=1}^{\ell(p)} q_{j}(p) p^{\mathbb{N}}$.

The picture for the general case is similar.

In the next three sections we look more carefully at the local factors.

\section{The local factors of the zeta function: the factor at infinity}

Let $\mathbf{G}$ be a connected, simply connected, complex almost simple algebraic group and $G=\mathbf{G}(\mathbb{C})$. As before $\mathcal{Z}_{G}(s)$ is the zeta function counting the rational representations of $G$. For example $\mathcal{Z}_{\mathrm{SL}_{2}(\mathbb{C})}(s)=\zeta(s)$, the Riemann zeta function, since $\mathrm{SL}_{2}$ has a unique irreducible rational representation of each degree.

In general, the irreducible representations of $G$ are parametrized by their highest weights as follows: Let $\Phi$ be the root system of $\mathbf{G}$ and $\varpi_{1}, \ldots, \varpi_{r}$ the fundamental weights. Write $\mathbb{N}=\{0,1,2, \ldots\}$, and for each $\left(a_{1}, \ldots, a_{r}\right) \in \mathbb{N}^{r}$ consider $\lambda=\sum a_{i} \varpi_{i}$. The irreducible representations $V_{\lambda}$ are parametrized by these weights $\lambda$. The Weyl dimension formula gives

$$
\operatorname{dim} V_{\lambda}=\prod_{\alpha \in \Phi^{+}} \frac{\alpha^{\vee}(\lambda+\rho)}{\alpha^{\vee}(\rho)}
$$

where $\Phi^{+}$is the set of positive roots, $\rho$ is half the sum of the roots in $\Phi^{+}$, and $\alpha^{\vee}$ is the dual root to $\alpha \in \Phi^{+}$. Note that $\prod_{\alpha \in \Phi^{+}} 1 / \alpha^{\vee}(\rho)$ is a constant depending only on $\mathbf{G}$ and not on $\lambda$, while the numerator $\prod_{\alpha \in \Phi^{+}} \alpha^{\vee}(\lambda+\rho)$ is a product of $\kappa=\left|\Phi^{+}\right|$linear functions in $a_{1}, \ldots, a_{r}$.

Theorem 5.1. The abscissa of convergence of $\mathcal{Z}_{\mathbf{G}(\mathbb{C})}(s)$ is equal to $r / \kappa$, where $r=\mathrm{rk} \mathbf{G}$ and $\kappa=\left|\Phi^{+}\right|$is the number of positive roots.

Proof. The description above implies that

$$
\mathcal{Z}_{G}(s)=\sum_{a_{1}=0}^{\infty} \cdots \sum_{a_{r}=0}^{\infty}\left(\operatorname{dim} V_{a_{1} \varpi_{1}+\cdots+a_{r} \varpi_{r}}\right)^{-s} .
$$

Thus we have a question of the following type: Given an $r \times \kappa$ matrix $b_{i j}$ of non-negative integers and a vector $c_{j}$ of positive integers, what is the abscissa of convergence of the Dirichlet series

$$
\sum_{a_{1}=0}^{\infty} \cdots \sum_{a_{r}=0}^{\infty}\left\{\prod_{j=1}^{\kappa}\left(b_{1 j} a_{1}+\cdots+b_{r j} a_{r}+c_{j}\right)\right\}^{-s}
$$


If we focus attention on the cube

$$
\left\{\left(a_{1}, \ldots, a_{r}\right) \mid 0 \leq a_{1}, \ldots, a_{r}<N\right\}
$$

we see that a typical term in this part of the sum is of size $O\left(\left(N^{\kappa}\right)^{-s}\right)$. Since there are $N^{r}$ such terms, one might guess that the abscissa of convergence corresponds to the real value $s$ for which $\left(N^{u}\right)^{-s}$ is comparable to the reciprocal of $N^{r}$, i.e. $s=r / \kappa$. For generic choices of the matrix $b_{i j}$, this turns out to be right. On the other hand, there may be subsets of the cube of substantial size for which the product of the sums $b_{1 j} a_{1}+\cdots+b_{r, j}+c_{j}$ is much smaller than $N^{\kappa}$. This happens if $\left(a_{1}, \ldots, a_{r}\right)$ lies near many of the hyperplanes $H_{j}: b_{1 j} x_{1}+\cdots+b_{r j} x_{j}=0$. (In our examples, these $H_{j}$ are precisely the walls of the Weyl chambers.)

To see how this can work, consider the series

$$
\sum_{a=0}^{\infty} \sum_{b=0}^{\infty} \sum_{c=0}^{\infty}((a+1)(a+b+1)(a+2 b+1)(c+1))^{-s} .
$$

If we consider only the $N$ terms with $a=b=0$, we obtain the Riemann zeta function, which diverges at $s=1$, where our naive guess gave convergence for $\mathfrak{R}(s)>3 / 4$. The problem is that three of the four rows of our matrix of coefficients lie in a two-dimensional subspace. In order to compute the abscissa of convergence in any particular case, we need to examine both the generic behavior on cubes $[0, N-1]^{r}$ and also behavior near the $H_{j}$. In fact, we may need to consider cases in which the index is near several $H_{j}$ but much nearer to some than to others. In the proof below, all of this is handled by a combinatorial strategy that breaks up $[0, N-1]^{r}$ into subsets according, roughly, to an integer vector which approximates the vector of the logarithms of the distances of an index $\left(a_{1}, \ldots, a_{r}\right)$ from each of the $H_{j}$.

We begin, though, with the easy direction, proving that $\mathcal{Z}_{G}(s)$ diverges for $s=r / \kappa$. If for $\lambda=\left(a_{1}, \ldots, a_{r}\right)$ and $m>0$, we have $a_{i} \leq m$ for every $i=1, \ldots, r$, then $\operatorname{dim} V_{\lambda} \leq c_{0} m^{\kappa}$ for some absolute constant $c_{0}$ depending only on $\mathbf{G}$ (since, as mentioned above, the numerator of $\operatorname{dim} V_{\lambda}$ is a product of $\kappa$ linear functions of the coefficients $a_{i}$ ). Thus $\left(\operatorname{dim} V_{\lambda}\right)^{-r / \kappa} \geq c_{1} m^{-r}$ for some constant $c_{1}>0$. Look now at the partial sums $S_{j}$ taken over all $\lambda=\left(a_{1}, \ldots, a_{r}\right)$ with $2^{j}<a_{i} \leq 2^{j+1}$. As there are $\left(2^{j+1}-2^{j}\right)^{r}=2^{j r}$ summands, and each of them contributes at least $c_{1}\left(2^{j+1}\right)^{-r}$, we have $S_{j} \geq c_{1} / 2^{r}$. The sets $S_{j}$ are disjoint so $\mathcal{Z}_{G}(r / \kappa) \geq \sum_{j=1}^{\infty} c_{1} / 2^{r}=\infty$.

We have now to prove that for every $s>r / \kappa, \mathcal{Z}_{G}(s)$ converges. For each $j \in \mathbb{N}$, let

$$
\Psi_{j}(\lambda):=\Phi \cap \operatorname{Span}_{\mathbb{R}}\left\{\alpha \in \Phi|| \alpha^{\vee}(\lambda+\rho) \mid<e^{j}\right\} .
$$

It is not difficult to check that $\Psi_{j}(\lambda)$ is itself a root system (reduced but not necessarily irreducible). Moreover, we clearly have

$$
\Psi_{1}(\lambda) \subseteq \Psi_{2}(\lambda) \subseteq \cdots
$$

and the sequence stabilizes at $\Phi$. 
Now, if $\alpha \in \Psi_{j+1}(\lambda) \backslash \Psi_{j}(\lambda)$ then $\log \left|\alpha^{\vee}(\lambda+\rho)\right|=j+O(1)$ and so

$$
\begin{aligned}
\log \operatorname{dim} V_{\lambda} & =\sum_{\alpha \in \Phi^{+}} \log \alpha^{\vee}(\lambda+\rho)+O(1) \\
& =\sum_{\alpha \in \Phi^{+}} \sum_{j=1}^{\infty} \eta(\alpha, j)+O(1) \text { where } \eta(\alpha, j)= \begin{cases}1, & \alpha \notin \Psi_{j}(\lambda), \\
0, & \alpha \in \Psi_{j}(\lambda) .\end{cases}
\end{aligned}
$$

The last sum is equal (up to a constant depending on $\Phi$ but not on $\lambda$ ) to $\sum_{i=1}^{\infty}\left(\left|\Phi^{+}\right|-\left|\Psi_{i}(\lambda)^{+}\right|\right)+O(1)$.

Let us now evaluate $\mathcal{Z}_{G}(s)$ for $s=r / \kappa+\epsilon$, for a fixed $\epsilon>0$. Every $\lambda$ gives rise to a sequence of root subsystems

$$
\Psi_{1}(\lambda) \subseteq \cdots \subseteq \Psi_{\ell}(\lambda)=\Phi .
$$

This is an increasing sequence but with possible repetitions. We will sum on $\lambda$ (and hence on these sequences) according to the subsequence which omits the repetitions. So we sum over all possible strictly increasing sequences of subsystems

$$
\Phi_{1} \subsetneq \cdots \subsetneq \Phi_{k}=\Phi
$$

Note that $k \leq r$ ( since $\operatorname{dim} \operatorname{Span} \Phi=r$ ). A sequence of type (5.2) determines (and is determined by) a sequence of type (5.3) together with a sequence of positive integers $b_{1}<\cdots<b_{k}$ such that

$$
\left\{\begin{array}{l}
\Psi_{1}(\lambda)=\cdots=\Psi_{b_{1}-1}(\lambda)=\emptyset \\
\Psi_{b_{1}}(\lambda)=\cdots=\Psi_{b_{2}-1}(\lambda)=\Phi_{1} \\
\Psi_{b_{2}}(\lambda)=\cdots=\Psi_{b_{3}-1}(\lambda)=\Phi_{2} \\
\ldots \ldots \ldots \ldots \ldots \ldots \ldots \ldots
\end{array}\right.
$$

Choose now a basis $\left\{\alpha_{1}, \ldots, \alpha_{r}\right\}$ for $\Phi$ such that the first $c_{1}$ vectors span the space $\operatorname{Span}\left(\Phi_{1}\right)$, the first $c_{2} \operatorname{span} \operatorname{Span}\left(\Phi_{2}\right)$ etc. This implies that for some constant $\delta_{1} \geq 1$,

$$
0<\alpha_{i}^{\vee}(\lambda+\rho) \leq \delta_{1} e^{b_{j}} \quad \forall i \leq c_{j}, i=1, \ldots, k .
$$

Now, given $\Phi_{1} \subsetneq \cdots \subsetneq \Phi_{k}$ we will sum over all possible sequences $1 \leq b_{1}<\cdots$ $<b_{k}$. We claim next that the number of dominant weights giving rise to a particular pair of sequences $\Phi_{1} \subsetneq \cdots \subsetneq \Phi_{k}$ and $b_{1}<\cdots<b_{k}$ is bounded above by a constant $\delta_{2}$ times

$$
\exp \left(b_{1} \operatorname{rk} \Phi_{1}+b_{2}\left(\operatorname{rk} \Phi_{2}-\operatorname{rk} \Phi_{1}\right)+\cdots+b_{k}\left(\operatorname{rk} \Phi_{k}-\operatorname{rk} \Phi_{k-1}\right)\right)
$$

To see this, observe that the map

$$
D: \lambda \mapsto\left(\alpha_{1}^{\vee}(\lambda), \ldots, \alpha_{r}^{\vee}(\lambda)\right)
$$

is an injective linear transformation from $\mathbb{N}^{r}$ (identified with the set of dominant weights via the map $\left.\left(a_{1}, \ldots, a_{r}\right) \mapsto \lambda=\sum_{i=1}^{r} a_{i} \varpi_{i}\right)$ to $\mathbb{N}^{r}$. The map

$$
\lambda \mapsto\left(\alpha_{1}^{\vee}(\lambda+\rho), \ldots, \alpha_{r}^{\vee}(\lambda+\rho)\right)
$$


is therefore an injective affine map. We need to bound the size of the set of all $\lambda$ in $\mathbb{N}^{r}$ which give rise to $\Phi_{1} \subsetneq \cdots \subsetneq \Phi_{k}$ and $b_{1}<\cdots<b_{k}$. Each such $\lambda$ satisfies all the inequalities of 5.5 . Since det $D$ is a constant, their number is indeed bounded by a constant $\delta_{2}$ times 5.6.

Finally, for each $\lambda$ the contribution of $V_{\lambda}$ to $\mathcal{Z}_{G}(r / \kappa+\epsilon)$ is bounded above by some constant $\delta_{3}$ times

$$
\exp \left(-\left(\frac{r}{\kappa}+\epsilon\right)\left(b_{1}\left|\Phi_{1}^{+}\right|+b_{2}\left(\left|\Phi_{2}^{+}\right|-\left|\Phi_{1}^{+}\right|\right)+\cdots+b_{k}\left(\left|\Phi_{k}^{+}\right|-\left|\Phi_{k-1}^{+}\right|\right)\right)\right) .
$$

To see this, note that 5.1 implies that $\log \operatorname{dim} V_{\lambda}=\sum_{i=1}^{k} b_{i}\left(\left|\Phi_{i}^{+}\right|-\left|\Phi_{i-1}^{+}\right|\right)+O(1)$ where $\Phi_{0}^{+}=\emptyset$.

Thus, for a suitable constant $\delta_{4}>0$, writing $\sum=\sum_{\emptyset=\Phi_{0} \subset \Phi_{1} \subset \cdots \subset \Phi_{k}=\Phi} \sum_{1 \leq b_{1}<\cdots<b_{k}}$, we have

$$
\begin{aligned}
& \mathcal{Z}_{G}\left(\frac{r}{\kappa}+\epsilon\right) \\
& \quad \leq \delta_{4} \sum \exp \left(\sum_{i=1}^{k} b_{i}\left(\operatorname{rk} \Phi_{i}-\operatorname{rk} \Phi_{i-1}\right)\right) \exp \left(-\left(\frac{r}{\kappa}+\epsilon\right) \sum_{i=1}^{k} b_{i}\left(\left|\Phi_{i}^{+}\right|-\left|\Phi_{i-1}^{+}\right|\right)\right) \\
& \quad=\delta_{4} \sum \exp \left(\sum_{i=1}^{k} b_{i}\left[\left(\operatorname{rk} \Phi_{i}-\operatorname{rk} \Phi_{i-1}\right)-\left(\frac{r}{\kappa}+\epsilon\right)\left(\left|\Phi_{i}^{+}\right|-\left|\Phi_{i-1}^{+}\right|\right)\right]\right) .
\end{aligned}
$$

To evaluate this sum we will use the following elementary convergence lemma:

Lemma. For constants $a_{1}, \ldots, a_{k} \in \mathbb{R}$ the series

$$
F_{k}\left(a_{1}, \ldots, a_{k}\right):=\sum_{1 \leq b_{1}<\cdots<b_{k}} \exp \left(\sum_{i=1}^{k} a_{i} b_{i}\right)
$$

converges if and only if

$$
a_{k}<0, \quad a_{k-1}+a_{k}<0, \ldots, a_{1}+\cdots+a_{k}<0 .
$$

Proof. We have

$$
\begin{aligned}
F_{k}\left(a_{1}, \ldots, a_{k}\right) & =\sum_{b_{1}=1}^{\infty} \exp \left(a_{1} b_{1}\right) \sum_{1+b_{1} \leq b_{2}<\cdots<b_{k}} \exp \left(a_{2} b_{2}+\cdots+a_{k} b_{k}\right) \\
& =\sum_{b_{1}=1}^{\infty} \exp \left(\left(a_{1}+\cdots+a_{k}\right) b_{1}\right) \sum_{1 \leq c_{2}<\cdots<c_{k}} \exp \left(a_{2} c_{2}+\cdots+a_{k} c_{k}\right) \\
& =\frac{\exp \left(a_{1}+\cdots+a_{k}\right)}{1-\exp \left(a_{1}+\cdots+a_{k}\right)} F_{k-1}\left(a_{2}, \ldots, a_{k}\right) \\
& =\prod_{n=1}^{k} \frac{\exp \left(a_{n}+\cdots+a_{k}\right)}{1-\exp \left(a_{n}+\cdots+a_{k}\right)}
\end{aligned}
$$

and the lemma follows. 
In our application $a_{i}=\left(\operatorname{rk} \Phi_{i}-\mathrm{rk} \Phi_{i-1}\right)-(r / \kappa+\epsilon)\left(\left|\Phi_{i}^{+}\right|-\left|\Phi_{i-1}^{+}\right|\right)$and hence for $i=1, \ldots, k$,

$$
a_{k}+a_{k-1}+\cdots+a_{i}=\left(\operatorname{rk} \Phi-\operatorname{rk} \Phi_{i-1}\right)-\left(\frac{r}{\kappa}+\epsilon\right)\left(\left|\Phi^{+}\right|-\left|\Phi_{i-1}^{+}\right|\right)
$$

(where $\Phi_{0}=\emptyset$ ).

We need to prove that (5.11) is less than 0 or equivalently

$$
\frac{\text { rk } \Phi-\operatorname{rk} \Phi_{i-1}}{\left|\Phi^{+}\right|-\left|\Phi_{i-1}^{+}\right|}<\frac{r}{\kappa}+\epsilon
$$

By inspection of all the pairs of irreducible root systems $\Phi_{i-1} \subset \Phi$, one sees that

$$
\frac{\operatorname{rk} \Phi_{i-1}}{\left|\Phi_{i-1}^{+}\right|} \geq \frac{\operatorname{rk} \Phi}{\left|\Phi^{+}\right|}=\frac{r}{\kappa}
$$

One can also give a conceptual proof of this inequality based on the observation that $r / \kappa=2 / h$ where $h$ is the Coxeter number. Now, if $\Phi_{i-1} \subset \Phi$, the Coxeter numbers satisfy $h_{i-1} \leq h$. This can be seen, for example, from the fact that the Coxeter number minus one is the largest exponent of the irreducible root system, and the fact that this is non-decreasing for inclusions of root systems can be deduced by comparing the orders of the corresponding Chevalley groups.

Now, if $\Phi_{i-1}$ is reducible, say $\Phi_{i-1}=\Phi_{i-1}^{\prime} \amalg \Phi_{i-1}^{\prime \prime}$, then

$$
\frac{\mathrm{rk} \Phi_{i-1}}{\left|\Phi_{i-1}^{+}\right|}=\frac{\mathrm{rk} \Phi_{i-1}^{\prime}+\mathrm{rk} \Phi_{i-1}^{\prime \prime}}{\left|\Phi_{i-1}^{\prime+}\right|+\left|\Phi_{i-1}^{\prime \prime}\right|} \geq \min \left\{\frac{\mathrm{rk} \Phi_{i-1}^{\prime}}{\left|\Phi_{i-1}^{+}\right|}, \frac{\mathrm{rk} \Phi_{i-1}^{\prime \prime}}{\left|\Phi_{i-1}^{\prime \prime}\right|}\right\} .
$$

The last inequality of (5.14) follows from the fact that if $a, b, c, d \in \mathbb{N}$ then we have $(a+c) /(b+d) \geq \min \{a / b, c / d\}$. It now follows that 5.13$)$ is true also if $\Phi_{i-1}$ is not necessarily irreducible.

Another elementary property of $a, b, c, d \in \mathbb{N}$ is that if $a \leq c, b \leq d$ and $a / b \geq c / d$ then $(c-a) /(d-b) \leq c / d$. Applying this for $a=\operatorname{rk} \Phi_{i-1}, b=\left|\Phi_{i-1}^{+}\right|, c=\operatorname{rk} \Phi$ and $d=\left|\Phi^{+}\right|$and using 5.13, we deduce that

$$
\frac{\operatorname{rk} \Phi-\operatorname{rk} \Phi_{i-1}}{\left|\Phi^{+}\right|-\left|\Phi_{i-1}^{+}\right|} \leq \frac{r}{\kappa}
$$

and hence (5.12) holds. This finishes the proof of Theorem 5.1

Remarks. (i) We have proved Theorem 5.1 in the simply connected case because this is the only case we need for the intended application, and because the parametrization of irreducible representations is slightly simpler in this case than in general. The theorem is true without this hypothesis, however, and the argument is unchanged except that instead of summing over $\mathbb{N}^{r}$, we sum over its intersection with some finite index subgroup of $\mathbb{Z}^{r}$.

(ii) As far as we know, the function $\zeta_{G}(s)$ first appeared in the literature in a paper of Witten [Wi], which discussed its values at positive even integers. If $\Sigma$ is a compact 
orientable surface of genus $g \geq 2, G^{c}$ is a compact form of $G$, and $E$ is a principal $G^{c}$ bundle over $\Sigma$, then $\zeta_{G}(s)$ arises in the computation of the volume of the moduli space $M$ of flat connections on $E$ up to gauge transformations. More precisely, $M$ has a natural symplectic structure $\omega$ and a natural volume form $\theta=\omega^{n} / n$ !, where $2 n=\operatorname{dim} M=$ $(2 g-2) \kappa, \kappa=\left|\Phi^{+}\right|$, and $\Phi$ is the root system of $\mathbf{G}$. As $\omega$ represents the first Chern class of a natural line bundle over $M$, the volume of $M$ with respect to $\theta$ is rational.

On the other hand, the same integral can be computed by means of a decomposition of $\Sigma$ into $2 g-2$ pairs of pants, and from this description it can be shown that up to a rational normalizing factor, $\int_{M} \theta$ is

$$
(2 \pi)^{-\operatorname{dim} M} \sum_{\lambda}\left(\operatorname{dim} V_{\lambda}\right)^{2-2 g}
$$

This shows that $\mathcal{Z}_{G}(s)=\sum_{\lambda}\left(\operatorname{dim} V_{\lambda}\right)^{-s}$ has the "zeta property" that its value at every positive even integer is a rational number times a suitable (integral) power of $\pi$.

We have no reason to believe this property is shared by our "global" zeta functions of arithmetic groups, but neither can we disprove it.

\section{The $p$-local factor}

We now shift our attention to the local factors at the finite primes, i.e., to $\mathcal{Z}_{L_{v}}(s)$ in the notation of Proposition 4.6 This is the representation zeta function of the group $L_{v}$ which is open in $\mathbf{G}\left(\mathcal{O}_{v}\right)$ and equal to $\mathbf{G}\left(\mathcal{O}_{v}\right)$ for almost all $v$.

When $\operatorname{char}(k)=0, L_{v}$ has an open uniform pro- $p$ subgroup (cf. [DDMS, Chapter 4]). An important result of A. Jaikin-Zapirain asserts:

Theorem 6.1 (Jaikin-Zapirain [J]a2]). Assume char $(k)=0$ and $p>2$, or if $p=2$, assume $L_{v}$ is uniform. Then $\mathcal{Z}_{L_{v}}(s)$ is a rational function in $p^{-s}$. More precisely, there exist natural numbers $k_{1}, \ldots, k_{t}$ and functions $f_{1}\left(p^{-s}\right), \ldots, f_{t}\left(p^{-s}\right)$ rational (with rational coefficients) in $p^{-s}$ such that

$$
\mathcal{Z}_{L_{v}}(s)=\sum_{i=1}^{t} k_{i}^{-s} f_{i}\left(p^{-s}\right)
$$

Problem 6.2. Does a similar result hold when $\operatorname{char}(k)>0$ ?

Theorem 6.1 is quite deep. It is proved by using Howe's interpretation of the Kirillov orbit method for uniform pro- $p$ groups [Ho]. This enabled Jaikin-Zapirain to present $\mathcal{Z}_{L_{v}}(s)$ as a $p$-adic integral and then to appeal to the work of Denef [De] on the rationality of such integrals.

Jaikin-Zapirain also made some explicit calculations. His main example is: 
Theorem 6.3. Let $\mathcal{O}_{v}$ be the ring of integers of a local field. Let $\mathcal{M}$ be its maximal ideal, $\mathbb{F}_{q}=\mathcal{O}_{v} / \mathcal{M}$ and $L_{v}=\operatorname{SL}_{2}\left(\mathcal{O}_{v}\right)$. If $q$ is odd, then

$$
\begin{aligned}
\mathcal{Z}_{L_{v}}(s)= & 1+q^{-s}+\frac{q-3}{2}(q+1)^{-s}+2\left(\frac{q+1}{2}\right)^{-s}+\frac{q-1}{2}(q-1)^{-s} \\
& +2\left(\frac{q-1}{2}\right)^{-s}+\frac{4 q\left(\frac{q^{2}-1}{2}\right)^{-s}+\frac{q^{2}-1}{2}\left(q^{2}-q\right)^{-s}+\frac{(q-1)^{2}}{2}\left(q^{2}+q\right)^{-s}}{1-q^{-s+1}}
\end{aligned}
$$

The reader can immediately see that the function depends only on $q$ and not on $\mathcal{O}_{v}$ and the abscissa of convergence of $L_{v}$ is always $\rho\left(L_{v}\right)=1$ independently of $q$ and $\mathcal{O}_{v}$ (see also Proposition 10.2 below). This is especially interesting since for $\mathbf{G}=\mathrm{SL}_{2}, r=\operatorname{rk} \mathbf{G}=1$ and $\left|\Phi^{+}\right|=1$, so $r / \kappa=1$.

Now we consider the general situation. Let $K$ be a non-archimedean local field and $\mathbf{G}$ an absolutely almost simple algebraic group over $K$. Fix a $K$-embedding of $\mathbf{G}$ in $\mathrm{GL}_{n}$ for some $n$, and let $U=\mathbf{G}(K) \cap \mathrm{GL}_{n}(\mathcal{O})$ where $\mathcal{O}$ is the ring of integers of $K$. We consider what can be said in general about $\rho(U)$.

Let $\pi$ be a uniformizer of $\mathcal{O}, q=|\mathcal{O} / \pi \mathcal{O}|$, and

$$
U_{k}=\operatorname{ker}\left(U \rightarrow \mathrm{GL}_{n}\left(\mathcal{O} / \pi^{k} \mathcal{O}\right)\right)
$$

Definition 6.4. (i) For a finite group $H$ we denote by $\bar{\gamma}(H)$ the number of its conjugacy classes.

(ii) We define $\gamma(U)$ (which may, a priori, depend on the embedding of $U$ in $\mathrm{GL}_{n}(\mathcal{O})$ ) as follows:

$$
\gamma=\gamma(U)=\limsup _{k \rightarrow \infty} \frac{\log _{q} \bar{\gamma}\left(U / U_{k}\right)}{k}
$$

Proposition 6.5. Let $\delta=\operatorname{dim}(\mathbf{G})$. Then

$$
\rho(U) \geq \frac{2 \gamma}{\delta-\gamma} .
$$

In other words: if $\mu=\gamma / \delta$ then $\rho(U) \geq 2 \mu /(1-\mu)$.

Proof. The quotient $U / U_{k}$ is of order approximately (up to a multiplicative constant) $q^{\delta k}$ and has approximately $q^{\gamma k}$ representations. If $q^{a k}$ is the median value of the degrees of these representations, then $\frac{1}{2} q^{\gamma k} \cdot\left(q^{a k}\right)^{2} \leq q^{\delta k}$. Hence $\gamma+2 a \leq \delta+o(1)$ as $k \rightarrow \infty$, i.e. $a \leq(\delta-\gamma) / 2$. This means that $U$ has at least $\frac{1}{2} q^{\gamma k}$ irreducible representations of degree at most $q^{\frac{1}{2}(\delta-\gamma) k}$. Hence $\rho(U) \geq 2 \gamma /(\delta-\gamma)$ as claimed.

Proposition 6.6. Let $K$ be a non-archimedean local field, $\mathbf{G}$ an almost $K$-simple algebraic group and $U$ an open compact subgroup of $\mathbf{G}(K)$. Then $\rho(U) \geq r / \kappa$ where $r$ is the absolute rank of $\mathbf{G}, \kappa=\left|\Phi^{+}\right|$, and $\Phi^{+}$is the set of positive roots in the absolute root system of $\mathbf{G}$. 
Proof. Fix an embedding $\mathbf{G} \hookrightarrow \mathrm{GL}_{n}$. Then $U\left(\subset \mathbf{G}(K) \subset \mathrm{GL}_{n}(K)\right)$ is commensurable with $\mathbf{G}(\mathcal{O}):=\mathbf{G}(K) \cap \mathrm{GL}_{n}(\mathcal{O})$ where $\mathcal{O}$ is the ring of integers of $K$. By Corollary 4.5, we can replace $U$ by $\mathbf{G}(\mathcal{O})$. Let $U_{k}=\operatorname{ker}\left(U \rightarrow \mathrm{GL}_{r}\left(\mathcal{O} / \pi^{k} \mathcal{O}\right)\right.$ ), where $\pi$ is a uniformizer of $\mathcal{O}$. Now, $\left[U: U_{k}\right]$, is of order approximately (up to a bounded multiplicative constant) $q^{k \operatorname{dim} \mathbf{G}}$ where $q=[\mathcal{O}: \pi \mathcal{O}]$. Let $\mathbf{T}$ be a maximal torus of $\mathbf{G}$. Then $\mathbf{T}(K) \cap U$ is a compact open subgroup of $\mathbf{T}(K)$ of dimension $r$. Its projection in $U / U_{k}$, denoted $\mathbf{T}\left(\mathcal{O} / \pi^{k} \mathcal{O}\right)$, is of order approximately $q^{k r}$. Fix a maximal torus in $\mathrm{GL}_{n}$ and let $L$ be a finite extension of $K$ over which this torus splits; let $\mathcal{O}_{L}$ denote the ring of integers in $L$. We can regard $U / U_{k}$ and its subgroup $\mathbf{T}\left(\mathcal{O} / \pi^{k} \mathcal{O}\right)$ as subgroups of $\mathrm{GL}_{n}\left(\mathcal{O}_{L} / \pi^{k} \mathcal{O}_{L}\right)$, and as such, the latter group can be conjugated into a diagonal subgroup.

We claim that for any local ring $(A, \mathfrak{m})$, two diagonal elements of $\mathrm{GL}_{n}(A)$ are conjugate if and only if their entries are the same up to order. To prove this, we give a basisindependent characterization of the multiplicity of an "eigenvalue" $\lambda \in A$ of a diagonalizable $A$-linear map $T$ from a rank- $n$ free $A$-module to itself. Namely, the multiplicity of $\lambda$ is the $(A / \mathfrak{m})$-dimension of the image of $\operatorname{ker}(T-\lambda \mathrm{Id}) \subset A^{n}$ in $(A / \mathfrak{m})^{n}$. We remark that this property is not true for general commutative rings. For instance, if $e$ is an idempotent, then

$$
\left(\begin{array}{cc}
e & e-1 \\
1-e & e
\end{array}\right)\left(\begin{array}{ll}
1 & 0 \\
0 & 0
\end{array}\right)\left(\begin{array}{cc}
e & e-1 \\
1-e & e
\end{array}\right)^{-1}=\left(\begin{array}{cc}
e & 0 \\
0 & 1-e
\end{array}\right) .
$$

As $\mathcal{O}_{L} / \pi^{k} \mathcal{O}_{L}$ is local, it follows that an element $x \in \mathbf{T}\left(\mathcal{O} / \pi^{k} \mathcal{O}\right)$ is conjugate to at most $n$ ! elements of $\mathbf{T}\left(\mathcal{O} / \pi^{k} \mathcal{O}\right)$ within $\mathrm{GL}_{n}\left(\mathcal{O}_{L} / \pi^{k} \mathcal{O}_{L}\right)$ and therefore, a fortiori, within $U / U_{k}$. This shows that $U / U_{k}$ has at least $c q^{k r}$ different conjugacy classes, for some $c>0$ which does not depend on $k$, and hence this number of different representations. By Proposition 6.5. $\rho(U) \geq r / \kappa$ as claimed.

\section{The $p$-local factor: anisotropic groups}

In this section we consider another class of examples for which $\rho=r / \kappa$, namely the anisotropic groups over local fields $K$ in characteristic zero. Let $D$ be a division algebra over $K$ of degree $d$ and $\mathbf{G}^{\prime}=\mathrm{SL}_{1}(D)$ the $K$-algebraic group of elements of $D$ of norm one. Thus $\mathbf{G}^{\prime}(K)$ is a compact virtually pro- $p$ group. This is a $K$-form of $\mathbf{G}=\mathrm{SL}_{d}$, i.e. over $\bar{K}$, the algebraic group $\mathrm{SL}_{1}(D)$ is isomorphic to $\mathrm{SL}_{d}$. Thus while $\operatorname{rk}_{K}\left(\mathbf{G}^{\prime}\right)=0$, the absolute rank of $\mathbf{G}^{\prime}$ is $d-1$ and the absolute root system is that of $\mathrm{SL}_{d}$. In particular,

$$
\left|\Phi^{+}\right|=\frac{d^{2}-d}{2} \text { and } \frac{r}{\kappa}=\frac{d-1}{\left(d^{2}-d\right) / 2}=\frac{2}{d} .
$$

Theorem 7.1. If $\operatorname{char}(K)=0$, then $\rho\left(\mathbf{G}^{\prime}(K)\right)=2 / d$.

Remark. We cannot prove this in positive characteristic but see Theorem 7.3 below.

Before starting the proof of Theorem 7.1 let us give a "linear algebra" lemma to be used in the proof. 
Lemma 7.2. Let $K \subset L$ be an extension of local fields with rings of integers $\mathcal{O}_{K}$ and $\mathcal{O}_{L}$. Let $\pi$ be a uniformizer of $K$ and $r \in \mathbb{N}$.

(i) If $T: \mathcal{O}_{K}^{n} \rightarrow \mathcal{O}_{K}^{n}$ is an injective $\mathcal{O}_{K}$-linear map, then

$\left|\operatorname{ker} T \otimes\left(\mathcal{O}_{L} / \pi^{r} \mathcal{O}_{L}\right)\right|=\left|\operatorname{cok} T \otimes\left(\mathcal{O}_{L} / \pi^{r} \mathcal{O}_{L}\right)\right|$

$$
=\left|\operatorname{ker} T \otimes\left(\mathcal{O}_{K} / \pi^{r} \mathcal{O}_{K}\right)\right|^{[L: K]}=\left|\operatorname{cok} T \otimes\left(\mathcal{O}_{K} / \pi^{r} \mathcal{O}_{K}\right)\right|^{[L: K]} .
$$

(ii) If $U: \mathcal{O}_{L}^{n} \rightarrow \mathcal{O}_{L}^{n}$ is an injective $\mathcal{O}_{L}$-linear map and

$$
\Lambda=\left\{x \in \mathcal{O}_{K}^{n} \mid x \otimes 1 \in \operatorname{im} U\right\},
$$

then

$$
\begin{aligned}
\left|\operatorname{ker} U \otimes\left(\mathcal{O}_{L} / \pi^{r} \mathcal{O}_{L}\right)\right| & =\left|\operatorname{cok} U \otimes\left(\mathcal{O}_{L} / \pi^{r} \mathcal{O}_{L}\right)\right| \\
& \leq\left|\operatorname{ker}\left(\Lambda \otimes\left(\mathcal{O}_{K} / \pi^{r} \mathcal{O}_{K}\right) \rightarrow\left(\mathcal{O}_{K} / \pi^{r} \mathcal{O}_{K}\right)^{n}\right)\right|^{[L: K]} \\
& =\left|\operatorname{cok}\left(\Lambda \otimes\left(\mathcal{O}_{K} / \pi^{r} \mathcal{O}_{K}\right) \rightarrow\left(\mathcal{O}_{K} / \pi^{r} \mathcal{O}_{K}\right)^{n}\right)\right|^{[L: K]} .
\end{aligned}
$$

Proof. If $D$ and $C$ denote the kernel and cokernel of $T \otimes\left(\mathcal{O}_{K} / \pi^{r} \mathcal{O}_{K}\right)$, respectively, then $|D|=|C|$ since the two middle terms in

$$
0 \rightarrow D \rightarrow\left(\mathcal{O}_{K} / \pi^{r} \mathcal{O}_{K}\right)^{n} \rightarrow\left(\mathcal{O}_{K} / \pi^{r} \mathcal{O}_{K}\right)^{n} \rightarrow C \rightarrow 0
$$

have equal order.

Now, as $\mathcal{O}_{L}$ is free of rank $[L: K]$ over $\mathcal{O}_{K}$, it follows that $\mathcal{O}_{L} / \pi^{r} \mathcal{O}_{L}$ is free over $\mathcal{O}_{K} / \pi^{r} \mathcal{O}_{K}$, and tensoring with it we obtain

$$
0 \rightarrow D \otimes\left(\mathcal{O}_{L} / \pi^{r} \mathcal{O}_{L}\right) \rightarrow\left(\mathcal{O}_{L} / \pi^{r} \mathcal{O}_{L}\right)^{n} \rightarrow\left(\mathcal{O}_{L} / \pi^{r} \mathcal{O}_{L}\right)^{n} \rightarrow C \otimes\left(\mathcal{O}_{L} / \pi^{r} \mathcal{O}_{L}\right) \rightarrow 0 .
$$

So,

$$
\left|D \otimes\left(\mathcal{O}_{L} / \pi^{r} \mathcal{O}_{L}\right)\right|=|D|^{[L: K]}=|C|^{[L: K]}=\left|C \otimes\left(\mathcal{O}_{L} / \pi^{r} \mathcal{O}_{L}\right)\right|,
$$

which gives (i).

For (ii), let $I$ denote the image of $U$. Let $S$ and $T$ denote the inclusion maps $I \hookrightarrow \mathcal{O}_{L}^{n}$ and $\Lambda \hookrightarrow \mathcal{O}_{K}^{n}$. As $T \otimes_{\mathcal{O}_{K}} \mathcal{O}_{L}$ factors through $S$, the image of $T \otimes_{\mathcal{O}_{K}}\left(\mathcal{O}_{L} / \pi^{r} \mathcal{O}_{L}\right)$ is contained in the image of $S \otimes_{\mathcal{O}_{L}}\left(\mathcal{O}_{L} / \pi^{r} \mathcal{O}_{L}\right)$. It follows that

$$
\left|\operatorname{cok} T \otimes_{\mathcal{O}_{K}}\left(\mathcal{O}_{L} / \pi^{r} \mathcal{O}_{L}\right)\right| \geq\left|\operatorname{cok} S \otimes_{\mathcal{O}_{L}}\left(\mathcal{O}_{L} / \pi^{r} \mathcal{O}_{L}\right)\right| .
$$

We conclude by applying part (i) to $T$.

Proof of Theorem 7.1. By Proposition 6.6, $\rho\left(\mathbf{G}^{\prime}(K)\right) \geq 2 / d$. It suffices, therefore, to prove only the upper bound.

Let us start by reviewing the "orbit method" classifying the representations of uniform pro- $p$ groups. Recall that a torsion-free pro- $p$ group $U$ is called uniform if $U^{p} \supseteq$ $[U, U]\left(U^{4} \supset[U, U]\right.$ if $\left.p=2\right)$. If $L$ is the Lie $\mathbb{Z}_{p}$-ring of $U$ (see [DDMS, §8.2]) then the irreducible representations of $U$ are in one-to-one correspondence with the orbits of homomorphisms $\varphi:(L,+) \rightarrow \mu_{p}$ (where $\mu_{p} \infty$ is the group of $p$-power roots of unity). 
By orbits here we mean orbits under the adjoint action of $U$ on $L$. Given such an $\varphi$, with orbit $[\varphi]$, the dimension of the corresponding representation is $|[\varphi]|^{1 / 2}$. (For a detailed description see $[\overline{\mathrm{Ho}}]$ and $[\overline{\mathrm{Ja} 2}]$.)

We can be more concrete in the setting of interest for the theorem. Let $\mathcal{O}$ be the ring of integers of $K, \pi$ a uniformizer of $\mathcal{O}, D_{0}$ the maximal $\mathcal{O}$-order of $D$ (which consists of all the elements of $D$ whose reduced norm is in $\mathcal{O}$ ), and $L$ the subspace of all the elements of $D_{0}$ of reduced trace 0 . The map $x \mapsto \exp (p x)$ from $L$ to $D$ takes $L$ into a uniform open subgroup of $\mathrm{SL}_{1}(D)$ which we will call $U$, whose Lie ring is $L$. Our goal is to prove that $\rho(U) \leq r / \kappa=2 / d$.

By the orbit method described above, we have to classify the characters of $L$. Let

$$
L^{*}=\left\{x \in D \mid \operatorname{Trd}_{D / K}(x)=0 \text { and } \operatorname{Tr}_{K / \mathbb{Q}_{p}} \operatorname{Trd}_{D / K}(x L) \subseteq \mathbb{Z}_{p}\right\} .
$$

Given a pair $(x, k)$ with $x \in L^{*}$ and $k \in \mathbb{N}$, the map

$$
\begin{gathered}
L \rightarrow \mathbb{Z}_{p} \rightarrow \mathbb{Z}_{p} / p^{k} \mathbb{Z}_{p} \rightarrow \mu_{p^{k}} \rightarrow \mu_{p^{\infty}}, \\
y \mapsto m=\operatorname{Tr}_{K / \mathbb{Q}_{p}}\left(\operatorname{Trd}_{D / K}(x y)\right) \mapsto m\left(\bmod p^{k}\right) \mapsto e^{2 \pi i m / p^{k}},
\end{gathered}
$$

is a character of $L$. As the Killing form is non-degenerate all characters are obtained in this way. There are, though, two types of repetition:

(i) The pairs $(x, k)$ and $(p x, k+1)$ induce the same character.

(ii) If $x_{1} \equiv x_{2}\left(\bmod p^{k}\right)$ in $L^{*}$ then $\left(x_{1}, k\right)$ and $\left(x_{2}, k\right)$ induce the same character.

We should also consider a third kind of equivalence among pairs $(x, k)$ :

(iii) For every $u \in U,(x, k) \sim\left(x^{u}, k\right)$, where $x^{u}$ is the image of $x$ under the conjugation action of $u$.

We will denote the equivalence class of $(x, k)$ by $[x, k]$. So, there is a one-to-one correspondence between the irreducible representations of $U$ and the equivalence classes $[x, k]$. The representation space associated to $[x, k]$ will be denoted by $V_{[x, k]}$. Note that the equivalences (i) and (ii) preserve character, while (iii) varies character within an equivalence class. We will denote by $|[x, k]|$ the number of characters associated with the equivalence class $[x, k]$. The orbit method implies that

$$
\operatorname{dim} V_{[x, k]}=|[x, k]|^{1 / 2} .
$$

Note that $L^{*}$ contains $L$ as a subgroup of finite index, so by equivalence (i) we can always assume that $x \in L$. The size of the orbit $|[x, k]|$ is equal to the index of the centralizer of $\exp (p x)$ in $U / U\left(p^{k}\right)$ where $U\left(p^{k}\right)=\exp \left(p^{k+1} L\right)$.

The division algebra $D$ has finitely many maximal subfields $F_{1}, \ldots, F_{c}$ such that every $x \in D$ is conjugate to one of them in $D$. This implies by (iii) and by the fact that $U$ is mapped onto a finite index subgroup of $D^{*} / Z\left(D^{*}\right)$, that we can sum up only on $x \in F_{i}$, $i=1, \ldots, c$. It therefore suffices to treat the contribution of a single $F=F_{i}$. 
The intersection $F \cap D_{0}$ is an order in $F$, and in establishing an upper bound, we may count all $x \in \mathcal{O}_{F}$ with $\operatorname{Tr}_{F / K}(x)=0$, where $\mathcal{O}_{F}$ is the ring of integers of $F$. Denote by $\pi$ a uniformizer of $\mathcal{O}_{F}$.

Let $\iota_{1}, \ldots, \iota_{d}$ denote the embeddings of $F$ into $\overline{\mathbb{Q}}_{p}$, a fixed algebraic closure of $\mathbb{Q}_{p}$. Let us denote by $\Phi$ the root system of type $A_{d-1}$ given via the standard basis $\left\{e_{1}, \ldots, e_{d}\right\}$ of $\mathbb{R}^{d}$ as

$$
\Phi=\left\{e_{s}-e_{t} \mid 1 \leq s, t \leq d, s \neq t\right\} .
$$

Given the pair $(x, k)$ as before, let

$$
\Psi_{i}(x, k)=\left\{e_{s}-e_{t} \in \Phi\left|p^{k}\right| \pi^{i}\left(\iota_{s}(x)-\iota_{t}(x)\right)\right\} .
$$

One can check that $\Psi_{i}(x, k)$ depends only on the equivalence class $[x, k]$, so we will denote it $\Psi_{i}[x, k]$. This is an increasing sequence

$$
\Psi_{1}[x, k] \subseteq \cdots \subseteq \Psi_{\ell}[x, k]=\Phi
$$

of root subsystems of $\Phi$, where $\ell=k e$ and $e$ is the ramification degree of $K$ over $\mathbb{Q}_{p}$.

The formal similarity with (5.2) deserves explanation or at least comment. The key is surely to be found in comparing the way in which the orbit method works in the two settings, $p$-adic analytic and compact real Lie groups. In the latter case, we can view dominant weights as integral $W$-orbits in $\mathfrak{t}^{*}$, where $\mathfrak{t}$ is a Cartan subalgebra of the Lie algebra. In each setting, the chain of root systems $\Psi_{i}$ characterizes the distances of a linear functional on a Cartan subalgebra to the walls of the Weyl chambers. To say more, we would need a unified way of viewing the Weyl dimension formula and (7.1). We do not know of such a way, but the similarities between the two proofs cry out for a unified treatment.

Claim 1. We have

$$
\operatorname{dim} V_{[x, k]}=\exp _{q}\left(\left(\sum_{i=1}^{\ell}\left|\Phi^{+} \backslash \Psi_{i}[x, k]\right|\right)-\left|\Phi^{+}\right|\right)
$$

where for $y \in \mathbb{R}, \exp _{q}(y)=q^{y}$.

Proof. We have

$$
\begin{aligned}
\operatorname{dim} V_{[x, k]} & =\left(\text { index of centralizer of } x \text { acting on } U / U\left(p^{k}\right)\right)^{1 / 2} \\
& \stackrel{(1)}{=} \exp _{q} \frac{1}{2}\left(\sum_{1 \leq s \neq t \leq d}\left(\min \left\{i \mid e_{s}-e_{t} \in \Psi_{i}[x, k]\right\}-1\right)\right) \\
& \stackrel{(2)}{=} \exp _{q} \frac{1}{2}\left(\left(\sum_{i=1}^{\ell}\left|\Phi \backslash \Psi_{i}[x, k]\right|\right)-|\Phi|\right) \\
& \stackrel{(3)}{=} \exp _{q}\left(\left(\sum_{i=1}^{\ell}\left|\Phi^{+} \backslash \Psi_{i}^{+}[x, k]\right|\right)-\left|\Phi^{+}\right|\right)
\end{aligned}
$$


Let us justify these equalities: (2) just reverses the order of summation between $i$ and $(s, t)$, while (3) follows from the central symmetry of all root systems involved. Equality (1) needs two remarks: First, as $x$-set: $U / U\left(p^{k}\right)$ is isomorphic to $L / p^{k} L$ via the map $\lambda \in L \mapsto \exp (p \lambda)$ which satisfies $\exp \left(x^{-1}(p \lambda) x\right)=x^{-1} \exp (p \lambda) x$.

Secondly, the size of the centralizer of $x$ acting on $U / U\left(p^{k}\right)$ is therefore the same as the kernel of $\operatorname{ad}(x)-\mathrm{Id}$ acting on $L / p^{k} L$. Lemma 7.2 implies that this kernel can be computed in a suitable extension which contains all the eigenvalues $\iota_{s}(x), 1 \leq s \leq d$. Then equality (1) becomes clear.

We now turn to the question of how many representations (i.e., equivalence classes $[x, k]$ ) give rise to a specified sequence $\left(\Psi_{1} \leq \cdots \leq \Psi_{\ell}=\Phi\right)$. The reader may note here that the structure of the proof is similar to that of Theorem 5.1 There is, however, a minor difference at this point. In the former proof every increasing chain actually occurs for some $\lambda$. Here, typically, many chains will not occur at all. This does not matter because at this point we are interested only in upper bounds.

Claim 2. The number of equivalence classes $[x, k]$ giving rise to a sequence $\Psi_{1} \leq \cdots \leq$ $\Psi_{\ell}=\Phi$ is bounded above by $\exp _{q}\left(\sum_{i=1}^{\ell}\left((d-1)-\operatorname{rk} \Psi_{i}\right)\right)$.

Proof. By Lemma 7.2 we may assume that all the eigenvalues of $x$ are in $\mathcal{O}$.

We know that $\Psi_{\ell}[x, k]=\Phi$ and the question is for how many $x$ 's (counted mod $\left.p^{k}\right)$, $\Psi_{\ell-1}[x, k]=\Psi_{\ell-1}$ etc.

For a fixed reduction of $x\left(\bmod \pi^{i-1}\right)$ we would like to count the number of possibilities modulo $\pi^{i}$. This means counting vectors $\left(\iota_{1}(x), \ldots, \iota_{d}(x)\right)$ subject to two additional conditions:

(i) trace $=\sum_{j=1}^{d} \iota_{j}(x)=0$,

(ii) $\iota_{s}(x) \equiv \iota_{t}(x)\left(\bmod \pi^{i}\right)$ for all $(s, t)$ such that $e_{s}-e_{t} \in \Psi_{\ell-i}$.

The dimension of the affine space of solutions to (ii) compatible with the specified reduction of $x\left(\bmod \pi^{i-1}\right)$ equals $d-\operatorname{rk} \Psi_{\ell-i}$ and condition (i) leads to $(d-1)-\operatorname{rk} \Psi_{\ell-i}$. This proves Claim 2.

Theorem 7.1 follows now from Claims 1 and 2 by a computation identical to that carried out in the proof of Theorem 5.1 .

We can prove Theorem 7.1 only for characteristic 0 where the orbit method is available. For quaternion algebras, however, we can prove the analogous theorem in odd characteristic as well.

Theorem 7.3. Let $K$ be a non-archimedean local field not of characteristic $2, D$ a central simple algebra over $K$ of degree $2, D_{0}$ a maximal order of $K$, and $\mathrm{Nrd}: D^{\times} \rightarrow K^{\times}$the reduced norm. Then

$$
\rho\left(D_{0}^{\times} \cap \operatorname{ker} \mathrm{Nrd}\right)=1 .
$$


Proof. As in the proof of Theorem 7.1, we only need to establish the upper bound. Lacking both a logarithm map and a satisfactory version of the orbit method in general, we develop a crude substitute for each.

Let $\mathcal{O}$ be the ring of integers in $K, \pi$ a uniformizer, and $q$ the order of the residue field of $\mathcal{O}$. Let

$$
U_{i}=1+\pi^{i} \mathcal{O} \subset \mathcal{O}^{\times}, \quad H_{i}=1+\pi^{i} D_{0} \subset D_{0}^{\times}, \quad G_{i}=\operatorname{ker} N_{i},
$$

where $N_{i}: H_{i} \rightarrow U_{i}$ is the restriction of Nrd. If $m<n \leq 2 m$, there are natural isomorphisms

$$
e_{m, n}^{\mathcal{O}}: \mathcal{O} / \pi^{n-m} \mathcal{O} \rightarrow U_{m} / U_{n} \quad \text { and } \quad e_{m, n}^{D}: D_{0} / \pi^{n-m} D_{0} \rightarrow H_{m} / H_{n}
$$

defined by

$$
e_{m, n}^{\mathcal{O}}\left(a+\pi^{n-m} \mathcal{O}\right)=\left(1+a \pi^{m}\right) U_{n} \quad \text { and } \quad e_{m, n}^{D}\left(a+\pi^{n-m} D_{0}\right)=\left(1+a \pi^{m}\right) H_{n} .
$$

In particular, $\left|U_{m} / U_{m+1}\right|=q$ and $\left|H_{m} / H_{m+1}\right|=q^{4}$ and therefore

$$
\left|U_{m} / U_{n}\right|=q^{n-m}, \quad\left|H_{m} / H_{n}\right|=q^{4(n-m)} .
$$

The identity

$$
\operatorname{Nrd}(1+a)=(1+a)(1+\bar{a})=1+\operatorname{Trd}(a)+\operatorname{Nrd}(a)
$$

implies that the diagram

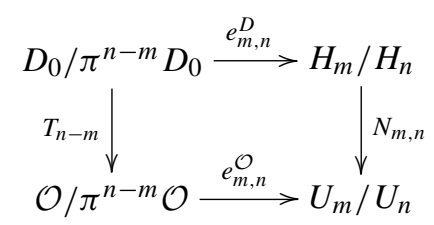

commutes, where $T_{n-m}$ and $N_{m, n}$ denote the maps induced by Trd and Nrd respectively.

Let $F \subset D$ be a separable quadratic extension of $K$. Then

$$
\operatorname{Trd}(F)=\operatorname{Tr}_{F / K}(F)=K,
$$

and $D_{0} \cap F$ is an open subring of $F$, so $\operatorname{Trd}\left(D_{0}\right) \supset \operatorname{Trd}\left(D_{0} \cap F\right)$ contains an open subgroup of $\mathcal{O}$. If $\pi^{c_{1}} \mathcal{O} \subset \operatorname{Trd}\left(D_{0}\right)$, then $\pi^{r+c_{1}} \mathcal{O} \subset \operatorname{Trd}\left(\pi^{r} D_{0}\right)$ for all non-negative integers $r$. This implies that if $r>c_{1}$, then

$$
\left(\operatorname{Nrd}\left(1+\pi^{r} D_{0}\right) \cap U_{r+c_{1}}\right) U_{r+c_{1}+1}=U_{r+c_{1}}
$$

and therefore by the completeness of $K$,

$$
\operatorname{Nrd}\left(H_{r}\right) \supset U_{r+c_{1}}
$$

for all $r>c_{1}$. This implies $\left|\operatorname{cok} N_{r}\right|<c_{2}$ for some constant $c_{2}$ independent of $r$. 
Let $L$ denote the Lie ring of elements of reduced trace 0 in $D_{0}$. Applying the snake lemma to

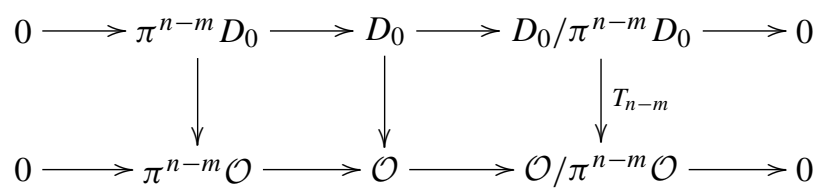

we see that

$$
\frac{\left|\operatorname{ker} T_{n-m}\right|}{\left|L / \pi^{n-m} L\right|} \leq c_{3} \quad \text { and } \quad\left|\operatorname{cok} T_{n-m}\right| \leq c_{3}
$$

for some constant $c_{3}$. When $m \leq n \leq 2 m$, we therefore find that

$$
\left|\operatorname{cok} N_{m, n}\right| \leq c_{3} \text {. }
$$

This inequality allows us to estimate $\left|G_{1} / G_{n}\right|$. Indeed,

$$
q^{3(n-m)} \leq\left|\operatorname{ker} N_{m, n}\right|=\frac{\left|H_{m} / H_{n}\right|\left|\operatorname{cok} N_{m, n}\right|}{\left|U_{m} / U_{n}\right|} \leq c_{3} q^{3(n-m)} .
$$

The snake lemma gives

$$
1 \rightarrow G_{m} / G_{n} \rightarrow \operatorname{ker} N_{m, n} \rightarrow \operatorname{cok} N_{n}
$$

and therefore

$$
\frac{\left|\operatorname{ker} N_{m, n}\right|}{\left|\operatorname{cok} N_{n}\right|} \leq\left|G_{m} / G_{n}\right|=\left|\operatorname{ker} N_{m} / \operatorname{ker} N_{n}\right| \leq\left|\operatorname{ker} N_{m, n}\right| \leq c_{3} q^{3(n-m)} .
$$

Applying this for $m=\lceil n / 2\rceil$ and iterating, we get

$$
\log \left|G_{1} / G_{m}\right|=3 m \log q+O(\log m)
$$

Next we directly compare $G_{m} / G_{n}$ and $L / \pi^{n-m} L$ for $c_{1} \leq m<n \leq 2 m$. The diagram (7.2) determines an $H_{1}$-equivariant isomorphism

$$
\operatorname{ker} T_{n-m} \stackrel{\sim}{\rightarrow} \operatorname{ker} N_{m, n} .
$$

We know that $L / \pi^{n-m} L$ and $G_{m} / G_{n}$ can each be realized as a subgroup of index $\leq c_{3}$ in $\operatorname{ker} N_{m, n}$. There is a natural relation between characters on the two subgroups according to which a character on the first group corresponds to a character on the second if each is the restriction of a common character on $\operatorname{ker} N_{m, n}$. Each character on either subgroup extends to at least 1 and at most $c_{3}$ characters on ker $N_{m, n}$. Each subgroup has a natural filtration, one arising from the filtration of $G_{m}$ by $G_{m+i}$ and the other from the filtration of $L$ by $\pi^{i} L$. These can be compared. For our purposes it is enough to note that the image of $G_{n-1} / G_{n}$ in $\operatorname{ker} N_{m, n}$ is contained in the image of $\pi^{n-m-1} L / \pi^{n-m} L$ in $\operatorname{ker} T_{n-m}$. It suffices to check that for every $a \in L$, we have $\operatorname{Nrd}\left(1+\pi^{n-1} a\right) \in \operatorname{Nrd}\left(H_{n}\right)$. This follows immediately from 7.3 since $\operatorname{Nrd}\left(1+\pi^{n-1} a\right) \in U_{2 n-2}$. 
Every continuous irreducible complex representation of $G_{1}$ is a representation of $G_{1} / G_{n}$ for some minimal $n$ which we call the level of the representation. We would like to find lower bounds for the dimensions of representations $V$ of level $n \geq 2 c_{1}$. Let $m=\lceil n / 2\rceil$, and consider the restriction of $V$ to the normal abelian subgroup $G_{m} / G_{n}$ of $G_{1} / G_{n}$. As $V^{G_{n-1} / G_{n}}$ is a $G_{1} / G_{n}$-subrepresentation of $V$, it must be trivial, since $V$ is of level $n$. Let $\chi_{0}$ denote a character of $G_{m} / G_{n}$ appearing in the restriction of $V$ to $G_{m} / G_{n}$. Every character in the $G_{1} / G_{n}$-orbit of $\chi_{0}$ in $\operatorname{Hom}\left(G_{m} / G_{n}, \mathbb{C}^{\times}\right)$appears in this restriction, so the dimension of $V$ is at least as large as the orbit of $\chi_{0}$, which is a character non-trivial on $G_{n-1} / G_{n}$. To find a lower bound for the size of this set, we need an upper bound on the size of the stabilizer of $\chi_{0}$.

Let $\phi_{0}$ denote a character of $L / \pi^{n-m} L$ which corresponds to $\chi_{0}$. The orbit size of $\phi_{0}$ differs from that of $\chi_{0}$ by at most a factor of $c_{3}$. As $\chi_{0}$ is non-trivial on $G_{n-1} / G_{n}, \phi_{0}$ is non-trivial on $\pi^{n-m-1} L / \pi^{n-m} L$. We therefore proceed by setting $r=n-m$ and finding a lower bound for the size of the orbit of

$$
\phi_{0} \in \operatorname{Hom}\left(L / \pi^{r} L, \mathbb{C}^{\times}\right) \backslash \operatorname{Hom}\left(L / \pi^{r-1} L, \mathbb{C}^{\times}\right) .
$$

To get this, we fix a character $\chi: K / \mathcal{O} \rightarrow \mathbb{C}^{\times}$such that $\chi\left(\pi^{-1}\right) \neq 1$. This gives a pairing

$$
\langle a, b\rangle=\chi(\operatorname{Trd}(a b))
$$

on $L \otimes K$. Let $L^{*}$ denote the kernel of $L$, i.e., the set of $b$ such that $\langle a, b\rangle=1$ for all $a \in L$. Thus $L^{*}$ is an $\mathcal{O}$-lattice in $L \otimes K$, and $L \subset L^{*}$. The map

$$
a \mapsto\left\langle\pi^{-r} a, x\right\rangle
$$

gives an isomorphism

$$
L^{*} / \pi^{r} L^{*} \rightarrow \operatorname{Hom}\left(L / \pi^{r} L, \mathbb{C}^{\times}\right) .
$$

As $L$ and $L^{*}$ are commensurable, the minimum orbit size of an element of $L^{*} / \pi^{r} L^{*}$ not divisible by $\pi$ and an element of $L / \pi^{r} L$ not divisible by $\pi$ differ by a bounded factor.

We are therefore led to the question of estimating the size of the stabilizer in $G_{1} / G_{r}$ of $x_{0} \in L / \pi^{r} L$ under the hypothesis $\pi \nmid x_{0}$. We begin by analyzing the set

$$
C_{x, r}=\left\{y \in D_{0} \mid x y-y x \in \pi^{r} D_{0}\right\}
$$

for a fixed $x$. We see that the set

$$
\begin{aligned}
S & =\left\{(x, y) \mid x \in L \backslash \pi L, y \in D_{0}, y \notin \operatorname{Span}_{K}\{1, x\}+\pi D_{0}\right\} \\
& =\left\{(x, y) \mid x \in L \backslash \pi L, y \in D_{0}, y \notin \operatorname{Span}_{\mathcal{O}}\{1, x\}+\pi D_{0}\right\}
\end{aligned}
$$

is compact and contains no pair of commuting elements. This is because in characteristic $\neq 2$, any trace-zero element of $M_{2}(\bar{K}) \supset D \supset D_{0}$ commutes only with linear combinations of itself and the identity. By compactness, there exists $c_{4}$ such that $x y-y x \notin \pi^{c_{4}} D_{0}$ for all $(x, y) \in S$. It follows that every $y \in B_{x, r}$ is congruent $\left(\bmod \pi^{r-c_{4}}\right)$ to an element of $\operatorname{Span}_{K}\{1, x\}$ and therefore congruent $\left(\bmod \pi^{r-c_{4}}\right)$ to an element of $\operatorname{Span}_{\mathcal{O}}\{1, x\}$. 
We count the number of elements of $y \in B_{x_{0}, r}$ such that $\operatorname{Nrd}(y)=1$ up to congruence $\left(\bmod \pi^{r-c_{4}}\right)$. Without loss of generality, we may assume that $y=u+v x_{0}$, where $u, v \in \mathcal{O}$. The norm condition asserts

$$
\operatorname{Nrd}\left(u+v x_{0}\right)=u^{2}+\operatorname{Nrd}\left(x_{0}\right) v^{2}=1
$$

so we count the number of solutions $(u, v)$ of this equation in the ring $\mathcal{O} / \pi^{r-c_{4}} \mathcal{O}$. The solution set is the union of solutions where $\pi \nmid u$ and solutions where $\pi \nmid v$. If $\left(u_{1}, v\right)$ and $\left(u_{2}, v\right)$ are solutions of the first kind, then

$$
\left(u_{1}+u_{2}\right)\left(u_{1}-u_{2}\right)=0, \quad \text { and } \quad\left(u_{1}+u_{2}\right)+\left(u_{1}-u_{2}\right)=2 u_{1} .
$$

As $u_{1}$ is a unit, this implies that the g.c.d. of $u_{1}+u_{2}$ and $u_{1}-u_{2}$ divides 2 . If 2 is $\pi^{c_{5}}$ times a unit, then

$$
u_{1} \equiv \pm u_{2}\left(\bmod \pi^{r-c_{4}-c_{5}}\right) .
$$

This gives at most $2 q^{c_{5}} q^{r-c_{4}}$ solutions where $\pi \nmid u$. The same argument applies when $\pi \nmid v$ as long as $\pi \mid \operatorname{Nrd}\left(x_{0}\right)$. If $\pi \nmid \operatorname{Nrd}\left(x_{0}\right)$, the same argument applies with the roles of $u$ and $v$ exchanged.

We conclude that

$$
\mid \operatorname{Stab}_{G_{1} / G_{r}}\left(x_{0} \mid \leq q^{2 c_{4}}\left(4 q^{r+c_{5}-c_{4}}\right)=c_{6} q^{r}\right.
$$

From this bound and (7.4), we can estimate the size of the orbit $O\left(x_{0}\right)$ :

$$
\log \left|O\left(x_{0}\right)\right|=2 r \log q+O(\log r)
$$

Thus, for all representations $V$ of level $n$,

$$
\log \operatorname{dim} V \geq 2 r \log q+O(\log r)
$$

As $r=n-m=[n / 2]$,

$$
\left|G_{1} / G_{n}\right|=6 r \log q+O(\log r)
$$

Since the sum of the squares of dimensions of all the representations of $G_{1} / G_{n}$ is $\left|G_{1} / G_{n}\right|$, the number of representations of level $n$ satisfies

$$
\log |\{V \mid \operatorname{level}(V)=n\}| \leq 2 r \log q+O(\log r) .
$$

Together, (7.5) and (7.6) imply the theorem. 


\section{The $p$-local factor: isotropic groups}

Theorems $5.1,7.1,7.3$ and 6.3 and Proposition 6.6 strongly suggest that $r / \kappa$ is always the abscissa of convergence. Our work on the subject was dominated for quite a long time by an effort to prove this. It turns out though that all these examples were misleading and in fact we have:

Theorem 8.1. If $K$ is a local non-archimedean field, $\mathbf{G}$ an isotropic almost simple $K$ group (i.e., $\operatorname{rk}_{K}(\mathbf{G}) \geq 1$ ) and $U$ an open compact subgroup of $\mathbf{G}(K)$, then $\rho(U) \geq 1 / 15$.

Remarks. (i) If $r=\operatorname{rk} \mathbf{G}$ goes to infinity then $r / \kappa \rightarrow 0$, thus Theorem 8.1 shows that $r / \kappa$ is usually not the abscissa of convergence. It still may be the right answer for groups of low $K$-rank.

(ii) The difference between isotropic and anisotropic groups is expressed by the fact that the first have non-trivial Bruhat-Tits building. The proof we give below does not refer to the buildings, but it seems that a better combinatorial understanding of them may lead to a more precise estimate on $\rho(U)$ (see $\S 11$ for more).

Proof. It suffices to treat the case of absolutely almost simple groups over $K$. Moreover, it suffices to treat one representative from every isogeny class. Tits [Ti] gives a full description of the classical absolutely simple groups over $K$. Note that in proving our theorem we may ignore the groups of type $G_{2}, F_{4}, E_{6}, E_{7}$ and $E_{8}$ as for these groups $r / \kappa \geq 1 / 15$, so our theorem follows from Proposition 6.6. Similarly, we can ignore forms of ${ }^{3} D_{4}$ and ${ }^{6} \mathrm{D}_{4}$. All the rest are given in [Ti, Table II] up to isogeny as groups of one of the following classical forms:

(a) $\mathrm{SL}_{m}(D)$ where $D$ is a central division algebra over $K$ of degree $d$. These are inner forms of $A_{n}$ for $n=m d-1$ (and we can assume $m \geq 2$ as we consider only isotropic groups).

(b) $\mathrm{SU}_{m}(L, f)$ where $L$ is a quadratic extension of $K$ and $f$ is a non-degenerate hermitian form of index $x \geq m / 2-1$. These are outer forms of $A_{m-1}$.

(c) $\mathrm{SO}_{m}(K, f)$, where $f$ is a quadratic form of index $x \geq m / 2-2$. These are inner forms of $B_{(m-1) / 2}$ if $m$ is odd, and they are forms of $D_{m / 2}$ if $m$ is even, outer or inner according to whether $m / 2-x$ is odd or even.

(d) $\mathrm{Sp}_{m}(K)$, where $m$ is even. These are the groups of type $C_{m / 2}$ and have index $x=$ $m / 2$.

(e) $\mathrm{SU}_{m}(D, f)$, where $D$ is the quaternion algebra over $K$ and $f$ is a non-degenerate antihermitian form of index $x \geq(m-1) / 2$. These are inner forms of $C_{m}$.

(f) $\mathrm{SU}_{m}(D, f)$, where $D$ is the quaternion algebra over $K$ and $f$ is a non-degenerate hermitian form of index $x \geq(m-3) / 2$. These are forms of $D_{m}$, outer or inner depending on $m-2 x$.

We will start with case (a). For simplicity we will assume first that $D=K$ and $m$ is even. We later remark how to modify the proof for the general case.

Let $X$ and $Z$ be diagonal $m / 2 \times m / 2$ matrices such that $\left(\begin{array}{ll}X & 0 \\ 0 & Z\end{array}\right)$ is a diagonal matrix in $\mathrm{SL}_{m}(\mathcal{O})$ which is regular and has trace 0 . For some $t$, all the diagonal entries are distinct $\left(\bmod \pi^{t}\right)$. 
We will consider the matrices $M_{Y}$ obtained by reducing $I+\pi^{k}\left(\begin{array}{ll}X & 0 \\ 0 & Z\end{array}\right)+\left(\begin{array}{ll}0 & Y \\ 0 & 0\end{array}\right)$ modulo $\pi^{3 k+2 t}$, where $X$ and $Z$ are fixed and $Y$ varies over the $q^{m^{2}(3 k+2 t) / 4}$ possibilities (mod $\left.\pi^{3 k+2 t}\right)$. Such matrices have determinant $1\left(\bmod \pi^{2 k}\right)$, so assuming that $k>t$, without sacrificing $\left(\bmod \pi^{t}\right)$ regularity, we can always modify $Z\left(\bmod \pi^{k}\right)$ to arrange that $M_{Y} \in$ $\mathrm{SL}_{m}\left(\mathcal{O} / \pi^{3 k+2 t} \mathcal{O}\right)$ for all $Y$.

Assume two such matrices $M_{Y}$ and $M_{Y^{\prime}}$ are conjugate. This means that there is an $m \times m$ matrix $\left(\begin{array}{ll}A & B \\ C & D\end{array}\right)$, where $A, B, C, D \in M_{m / 2}\left(\mathcal{O} / \pi^{3 k+2 t} \mathcal{O}\right), \operatorname{det}\left(\begin{array}{ll}A & B \\ C & D\end{array}\right)=1$ and

$$
\left(\begin{array}{ll}
A & B \\
C & D
\end{array}\right)\left(\begin{array}{cc}
\pi^{k} X & Y \\
0 & \pi^{k} Z
\end{array}\right)=\left(\begin{array}{cc}
\pi^{k} X & Y^{\prime} \\
0 & \pi^{k} Z
\end{array}\right)\left(\begin{array}{ll}
A & B \\
C & D
\end{array}\right) .
$$

From (8.1) we can deduce:

(i) Considering the lower left block,

$$
C X \equiv Z C\left(\bmod \pi^{2 k+2 t}\right),
$$

which implies $C \equiv 0\left(\bmod \pi^{2 k+t}\right)$ since the difference of any diagonal entry of $X$ and any diagonal entry of $Z$ cannot be divisible by $\pi^{t+1}$.

(ii) Considering the upper left block,

$$
\pi^{k} A X \equiv \pi^{k} X A+Y^{\prime} C\left(\bmod \pi^{3 k+2 t}\right) .
$$

From (i) we know that $C \equiv 0\left(\bmod \pi^{2 k+t}\right)$, so we get

$$
A X \equiv X A\left(\bmod \pi^{k+t}\right)
$$

which implies that $A$ is diagonal $\left(\bmod \pi^{k}\right)$ since the difference between two distinct diagonal entries of $A$ cannot be divisible by $\pi^{t+1}$.

(iii) Considering the lower right block,

$$
C Y+\pi^{k} D Z \equiv \pi^{k} Z D\left(\bmod \pi^{3 k}\right),
$$

and hence by (i),

$$
D Z \equiv Z D\left(\bmod \pi^{k+t}\right)
$$

and so $D$ is diagonal $\left(\bmod \pi^{k}\right)$.

(iv) From (ii) and (iii) and $\operatorname{det}\left(\begin{array}{cc}A & B \\ C & D\end{array}\right)=1$, we deduce that $A$ and $D$ are invertible $(\bmod \pi)$ (since $C \equiv 0(\bmod \pi)$ ) and hence also $\left(\bmod \pi^{k}\right)$.

(v) The upper right corner now gives

$$
A Y \equiv Y^{\prime} D\left(\bmod \pi^{k}\right) .
$$

So,

$$
A Y D^{-1} \equiv Y^{\prime}\left(\bmod \pi^{k}\right) .
$$


Let now

$$
\tilde{M}=\left\{M_{Y} \mid Y \in M_{m / 2}\left(\mathcal{O} / \pi^{3 k+2 t} \mathcal{O}\right)\right\} .
$$

Choose out of $\tilde{M}$ a set $M$ of $q^{m^{2} k / 4}$ representatives for the different possible images of $Y$ $\left(\bmod \pi^{k}\right)$.

Assertions (ii)-(v) imply that for a given such $M_{Y} \in M$, there are at most $q^{(2 \cdot m / 2-1) k}$ $=q^{(m-1) k}$ other elements of $M$ which are in the same conjugacy class. This implies that $M$ has representatives of at least $q^{\left(m^{2} / 4-m+1\right) k}$ different conjugacy classes. In particular, $\mathrm{SL}_{m}\left(\mathcal{O} / \pi^{3 k+2 t} \mathcal{O}\right)$ has at least $q^{\left(m^{2} / 4-m+1\right) k}$ conjugacy classes. So we have proved that

$$
\gamma\left(\mathrm{SL}_{m}(\mathcal{O})\right) \geq \frac{1}{3}\left(\frac{m^{2}}{4}-m+1\right)=\frac{1}{12}\left(m^{2}-4 m+4\right) .
$$

Proposition 6.5 now implies that

$$
\rho\left(\mathrm{SL}_{m}(\mathcal{O})\right) \geq \frac{\frac{1}{6}\left(m^{2}-4 m+4\right)}{m^{2}-1-\frac{1}{12}\left(m^{2}-4 m+4\right)} .
$$

This proves the theorem for every $m \geq 6$ even. For $m=4$ we can use Proposition 6.6

If we replace $K$ by $D$ (and $m$ is still even) the proof works as it stands (recall that $\mathrm{SL}_{m}(D)$ means the set of all $m \times m$ matrices over $D$ whose determinant, considered as an $m d \times m d$ matrix over $\bar{K}$, is one.) The only modification needed is when computing dimensions: the number of elements of $M$ is $q^{m^{2} d^{2} k / 4}$ and every element there can be conjugated to at most $q^{(m d-1) k}$ other elements of $M\left(\operatorname{since} \operatorname{rk}\left(\operatorname{SL}_{m}(D)\right)=m d-1\right.$.)

We deduce that for an open subgroup $U$ of $\operatorname{SL}_{m}(D), \gamma(U) \geq \frac{1}{3}\left(\frac{1}{4} m^{2} d^{2}-m d+1\right)$ and since $\operatorname{dim}_{k}\left(\operatorname{SL}_{m}(D)\right)=m^{2} d^{2}-1$ we have

$$
\rho(U) \geq \frac{\frac{2}{3}\left(\frac{1}{4} m^{2} d^{2}-m d+1\right)}{m^{2} d^{2}-1-\frac{1}{3}\left(\frac{1}{4} m^{2} d^{2}-m d+1\right)} .
$$

This proves the theorem for $m d \geq 6$. For $m d \leq 4$, we can use Proposition 6.6

Finally, for general $m$ we will write $m$ as $m=\left[\frac{m+1}{2}\right]+\left[\frac{m}{2}\right]$ and in the proof we will work with blocks of sizes $\left[\frac{m+1}{2}\right]$ and $\left[\frac{m}{2}\right]$. E.g., $X$ is an $\left[\frac{m+1}{2}\right] \times\left[\frac{m+1}{2}\right]$ matrix, $Y$ is $\left[\frac{m+1}{2}\right] \times\left[\frac{m}{2}\right], Z$ is $\left[\frac{m}{2}\right] \times\left[\frac{m}{2}\right]$ etc. The proof (for $K$ or $D$ ) carries over without any difficulty. The size of $M$ is then $q^{\left[\frac{m+1}{2}\right]\left[\frac{m}{2}\right] d^{2} k}$, and every element of it is conjugate to at most $q^{(m d-1) k}$ elements, so

$$
\gamma(U) \geq \frac{1}{3}\left(\left[\frac{m+1}{2}\right]\left[\frac{m}{2}\right] d^{2}-m d+1\right),
$$

and

$$
\rho(U) \geq \frac{\frac{2}{3}\left(\left[\frac{m+1}{2}\right]\left[\frac{m}{2}\right] d^{2}-m d+1\right)}{m^{2} d^{2}-1-\frac{1}{3}\left(\frac{1}{4} m^{2} d^{2}-m d+1\right)} .
$$


This time we can assume $m \geq 3$. For $m \geq 3$ odd, the only pairs $(m, d)$ for which this quantity is less than $1 / 15$ are $(3,1),(3,2)$, and $(5,1)$. For these cases we can use Proposition 6.6

We now turn to groups of type (b)-(f), i.e. $\mathrm{SU}_{m}(D, f)$ where $D$ is either $K, L$ or the quaternion algebra over $K$ and $f$ is a Hermitian or skew-Hermitian form on $W=D^{m}$. By definition of index, we can choose a basis

$$
e_{1}, \ldots, e_{x}, f_{1}, \ldots, f_{x}, g_{1}, \ldots, g_{s}
$$

with respect to which our form can be written in blocks of sizes $x, x$, and $m-2 x$ as follows:

$$
\left(\begin{array}{ccc}
0 & I & 0 \\
\pm I & 0 & 0 \\
0 & 0 & N
\end{array}\right)
$$

(Note that if $m=2 x$, the third block size is zero, so in fact we will have a $2 \times 2$ block matrix.)

For fixed $x \times x$ matrices $X$ and $Z$, we consider matrices of the form

$$
M_{Y}=I_{m}+\left(\begin{array}{ccc}
\pi^{k} X & Y & 0 \\
0 & \pi^{k} Z & 0 \\
0 & 0 & 0
\end{array}\right)
$$

If $X=Z=0$, the condition on $Y$ for this matrix to lie in $\mathrm{SU}_{m}(D, f)$ is $Y \pm \sigma(Y)=0$, where $\sigma$ is the involution (possibly trivial) defining the group. The number of distinct possibilities for $Y\left(\bmod \pi^{k}\right)$ is $q^{x^{2} k}, q^{\left(x^{2}-x\right) k / 2}, q^{\left(x^{2}+x\right) k / 2}, q^{\left(2 x^{2}+x\right) k}$, and $q^{\left(2 x^{2}-x\right) k}$ for cases (b), (c), (d), (e), and (f) respectively. By conjugation, we see that whenever $X$ and $Z$ are chosen so that $M_{0} \in \mathrm{SU}_{m}(D, f)$, the number of possible values of $Y\left(\bmod \pi^{k}\right)$ for which $M_{Y} \in \mathrm{SU}_{m}(D, f)$ is the same. We will fix $X, Z$ to be diagonal matrices which have all $2 x$ entries distinct $\left(\bmod \pi^{t}\right)$. For some value of $t$ depending only on $m$ and the order $q$ of the residue field of $K$, we can always do this.

If $M_{Y}$ and $M_{Y^{\prime}}$ are conjugate, we have a $\left(\bmod \pi^{3 k+2 t}\right)$ equality:

$$
\left(\begin{array}{ccc}
A & B & C \\
D & E & F \\
G & H & J
\end{array}\right)\left(\begin{array}{ccc}
I_{x}+X & Y & 0 \\
0 & I_{x}+Z & 0 \\
0 & 0 & I_{s}
\end{array}\right)=\left(\begin{array}{ccc}
I_{x}+X & Y^{\prime} & 0 \\
0 & I_{x}+Z & 0 \\
0 & 0 & I_{s}
\end{array}\right)\left(\begin{array}{ccc}
A & B & C \\
D & E & F \\
G & H & J
\end{array}\right)
$$

Imitating steps (i)-(v) above, we prove first that $D \equiv 0\left(\bmod \pi^{2 k+t}\right)$ and next that $A$ and $E$ are diagonal $\left(\bmod \pi^{k}\right)$. Finally, we conclude that there are at least $q^{n}$ distinct conjugacy classes, where $n$ is $x^{2} k-2 x k,\left(x^{2}-x\right) k / 2-x k,\left(x^{2}+x\right) k / 2-x k,\left(2 x^{2}+x\right) k-4 x k$, and $\left(2 x^{2}-x\right) k-4 x k$ for cases (b) through (f) respectively. Using the relation between $m$ and $x$, we conclude that 


$$
\rho(U) \geq \begin{cases}\frac{2 x^{2}-4 x}{(2 x+2)^{2}-1-\left(x^{2}-2 x\right)} & \text { in case (b), } \\ \frac{x^{2}-3 x}{(2 x+4)(2 x+3) / 2-\left(x^{2}-3 x\right) / 2} & \text { in case (c), } \\ \frac{x^{2}-x}{2 x(2 x+1) / 2-\left(x^{2}-x\right) / 2} & \text { in case (d), } \\ \frac{4 x^{2}+2 x}{(4 x+2)(4 x+1) / 2-\left(2 x^{2}+x\right)} & \text { in case (e), } \\ \frac{4 x^{2}-2 x}{(4 x+6)(4 x+5) / 2-\left(2 x^{2}-x\right)} & \text { in case (f). }\end{cases}
$$

In all cases, therefore,

$$
\rho(U) \geq \frac{2 x^{2}-6 x}{3 x^{2}+17 x+12},
$$

so $\rho(U)>1 / 15$ for $x \geq 5$. For $x \leq 4$, we have rank $\leq 11$, and therefore Coxeter number $\leq 30$. Thus, Proposition 6.6 covers all these cases.

Remarks. Theorems 7.1 and 8.1 show a dichotomy in the asymptotic behavior of $\rho(U)$ between isotropic and anisotropic groups. It should be noted, however, that the number $\rho(U)$ itself cannot distinguish between the two: For example, for a quaternion algebra $D$ over $K$ of characteristic zero, $\rho\left(\operatorname{SL}_{1}(D)\right)=1$ and at the same time $\rho\left(\operatorname{SL}_{2}(\mathcal{O})\right)=1$ when $\mathcal{O}$ is the ring of integers of $K$.

\section{Applications to general groups}

We can now apply the results of the previous section to prove the following theorem:

Theorem 9.1. If $\Gamma$ is a finitely generated infinite linear group over some field $F$ (or more generally, if $\Gamma$ is a finitely generated group with some homomorphism $\varphi: \Gamma \rightarrow \operatorname{GL}_{n}(F)$ with $\varphi(\Gamma)$ infinite) then $\rho(\Gamma) \geq 1 / 15$.

Proof. If $\Gamma$ is a quotient of $\Gamma_{1}$ then $0 \leq \rho(\Gamma) \leq \rho\left(\Gamma_{1}\right)$, so it suffices to prove the result for the case of a linear group $\Gamma$. Moreover, we can replace $F$ by the ring generated by the entries of the generators of $\Gamma$ to deduce that $\Gamma$ is inside $\mathrm{GL}_{n}(A)$ for some finitely generated subring $A$ of $F$. Let now $\mathbf{G}$ be the Zariski closure of $\Gamma$. If $\mathbf{G}$ is virtually solvable then $\Gamma$ has a finite index subgroup with an infinite abelianization. This implies that for some $\ell, \Gamma$ has infinitely many $\ell$-dimensional irreducible representations and so $\rho(\Gamma)$ $=\infty$ and we are done. So assume $\mathbf{G}$ is not virtually solvable and we can then replace $\mathbf{G}$ by its quotient modulo the solvable radical and replace $\Gamma$ by a finite index subgroup (using Corollary 4.5) to assume that $\mathbf{G}$ is semisimple, or even simple by taking a (non-trivial) simple quotient. 
Now, we specialize $A$ into a global field $k$, keeping $\Gamma$ non-virtually solvable. In fact, it was shown in [LaLu, Theorem 4.1] that this can be done keeping $\mathbf{G}$ as the Zariski closure.

So, altogether we can assume $\Gamma$ is a Zariski dense subgroup in $\mathbf{G}(k)$, where $\mathbf{G}$ is a simple $k$-group. Let $U_{v}$ denote the closure, in the $v$-adic topology, of $\Gamma$ in $\mathbf{G}\left(k_{v}\right)$ for some non-archimedean place $v$ for which $\mathbf{G}$ is isotropic over $k_{v}$ and that closure is compact. Note that all but finitely many $v$ satisfy each condition, so there is no difficulty in fixing $v$ satisfying both. By Pink's characterization of Zariski dense compact subgroups of semisimple groups over local fields [Pi], there exists a finite extension $k_{v}^{\prime}$ of $k_{v}$, a simply connected, almost simple algebraic group $\mathbf{G}^{\prime}$ over $k_{v}^{\prime}$, and a compact open subgroup $U_{v}^{\prime} \subset \mathbf{G}^{\prime}\left(k_{v}^{\prime}\right)$ such that $U_{v}$ is topologically isomorphic to the quotient of $U_{v}^{\prime}$ by its intersection with the center of $\mathbf{G}^{\prime}\left(k_{v}^{\prime}\right)$. Replacing $U_{v}^{\prime}$ with an open subgroup which meets that center only in the identity, we see that $U_{v}$ has an open subgroup which is topologically isomorphic to an open subgroup of the $k_{v}^{\prime}$ points of the almost simple algebraic group $\mathbf{G}^{\prime}$. Hence $\rho(\Gamma) \geq \rho\left(U_{v}\right) \geq 1 / 15$ by Theorem 8.1 .

We now show that Theorem 9.1 is not valid in general for finitely generated, residually finite groups. In fact, we can even prove:

Theorem 9.2. There exists a finitely generated, residually finite, infinite group $\Gamma$ with $\rho(\Gamma)=0$.

Proof. Let us recall first the result of Liebeck and Shalev counting representations of the alternating groups $A_{k}$.

Theorem 9.3 (Liebeck-Shalev [LiSh1]). For every $s>0, \lim _{k \rightarrow \infty} \mathcal{Z}_{A_{k}}(s)=1$ where as before $\mathcal{Z}_{A_{k}}(s)=\sum_{i=1}^{\infty} r_{i}\left(A_{k}\right) i^{-s}$.

This theorem can be converted to an explicit upper bound on representation growth, via the following lemma:

Lemma 9.4. If $G$ is a perfect finite group, $0<s<1$, and $\mathcal{Z}_{G}(s)<1+c$, then for every $n \in \mathbb{N}$, we have $R_{n}(G) \leq c n^{s}+1$.

Proof. As $G$ is perfect, $r_{1}(G)=1$. Then

$$
\left(R_{n}(G)-1\right) n^{-s}=\sum_{i=2}^{n} r_{i}(G) n^{-s} \leq \sum_{i=2}^{n} r_{i}(G) i^{-s} \leq c,
$$

which implies the lemma.

Let us now recall some results of Segal $[\bar{S}]$ : Let $\ell_{0}, \ell_{1}, \ell_{2}, \ldots$ be a sequence of positive integers. We construct, by induction, a sequence of finite groups $W_{r}$ as follows:

$$
W_{0}=A_{\ell_{0}}, W_{1}=A_{\ell_{1}}^{\ell_{0}} \rtimes W_{0}, W_{2}=A_{\ell_{2}}^{\ell_{0} \ell_{1}} \rtimes W_{1}, \ldots, W_{r}=A_{\ell_{r}}^{\ell_{0} \ldots \ell_{r-1}} \rtimes W_{r-1}, \ldots
$$

These are wreath products obtained as natural subgroups of the automorphism group of the rooted tree with degree $\ell_{0}$ at the origin and degree $\ell_{i}+1$ for all the vertices of level 
$i>0$ (i.e. of distance $i$ from the origin). See [S] for the detailed description. Let $W$ be the profinite group $W=\lim _{r} W_{r}$ with the obvious morphisms. It is also shown in [S] that $W$ contains a finitely generated dense subgroup $\Gamma$ whose profinite completion is isomorphic to $W$ via the natural map $\hat{\Gamma} \rightarrow W$ extending the embedding $\Gamma \hookrightarrow W$. It is easy to deduce that $\Gamma$ is not a linear group. Moreover, every representation of it factors through one of the $W_{r}$. Indeed, if $\Gamma$ had had an infinite non-virtually solvable representation then (by an application of strong approximation for linear groups [LS, pp. 389-407]) there would have been infinitely many simple groups of Lie type among the composition factors of $\hat{\Gamma}=W$. But as we know, all the composition factors of $W$ are alternating groups. Moreover, $W$ (and hence $\Gamma$ ) has the (FAb) property (i.e., every finite index subgroup has a finite abelianization) and so $\Gamma$ has no infinite virtually-solvable quotients either. Thus every representation factors through some $W_{r}$. Moreover, as the kernels $\operatorname{ker}\left(W \rightarrow W_{r}\right)$ are the only finite index normal subgroups of $W$, a representation of $W_{r}$ which does not factor through $W_{r-1}$ must be faithful.

Let us now choose a sequence $\ell_{0}, \ell_{1}, \ell_{2}, \ldots$ which grows sufficiently fast. More specifically, we want

$$
\frac{\log \left|W_{r-1}\right|}{\log \ell_{r}}<\frac{1}{r}
$$

and

$$
\mathcal{Z}_{A_{\ell_{r}}}\left(\frac{1}{r}\right)<1+\frac{1}{L_{r-1}}
$$

where $L_{r-1}=\ell_{0} \ell_{1} \cdots \ell_{r-1}$.

Note that since $\left|W_{r-1}\right|=\left(\frac{1}{2} \ell_{r-1} !\right)^{\ell_{0} \cdots \ell_{r-2}}\left|W_{r-2}\right|$, the order of $W_{r-1}$ depends only on $\ell_{0}, \ldots, \ell_{r-1}$, so we can choose $\ell_{r}$ large enough to satisfy 9.1 . Also as $\mathcal{Z}_{A_{k}}(1 / r) \rightarrow 1$ as $k \rightarrow \infty$, we can make sure that $\ell_{r}$ also satisfies $(9.2)$.

Given the sequence $\ell_{0}, \ell_{1}, \ell_{2}, \ldots$ let $W$ and $\Gamma$ be the groups as defined before with respect to this sequence. We have to bound $r_{n}(\Gamma)$.

So given $n \in \mathbb{N}$, let $r$ be the unique natural number for which $\ell_{r}-1 \leq n<\ell_{r+1}-1$. As $A_{\ell_{r+1}}$ is a subgroup of $W_{r+1}$ and every non-trivial representation of $A_{\ell_{r+1}}$ is of dimension at least $\ell_{r+1}-1$, all the $n$-dimensional representations of $\Gamma$ factor through $W_{r}$ for this $r$. By Proposition 4.4,

$$
R_{n}\left(W_{r}\right) \leq\left|W_{r-1}\right| R_{n}\left(A_{\ell_{r}}^{\ell_{0} \ldots \ell_{r-1}}\right)
$$

where $R_{n}$ is the number of all irreducible representations of dimension at most $n$. Thus

$$
\lim _{n \rightarrow \infty} \frac{\log R_{n}(\Gamma)}{\log n}=\lim _{n \rightarrow \infty} \frac{\log R_{n}\left(W_{r}\right)}{\log n} \leq \lim _{n \rightarrow \infty} \frac{\log \left|W_{r-1}\right|}{\log n}+\lim _{n \rightarrow \infty} \frac{\log R_{n}\left(A_{\ell_{r}}^{\ell_{0} \ldots \ell_{r-1}}\right)}{\log n}
$$

As $n \geq \ell_{r}-1$, 9.1) implies the first summand is zero.

For the second summand, note that for $L_{r-1}=\ell_{0} \cdots \ell_{r-1}$,

$$
\mathcal{Z}_{A_{\ell_{r}}^{L_{r-1}}}(s)=\mathcal{Z}_{A_{\ell_{r}}}(s)^{L_{r-1}}
$$


Thus by 9.2 we get

$$
\mathcal{Z}_{A_{\ell_{r}}^{L_{r-1}}}\left(\frac{1}{r}\right)<\left(1+\frac{1}{L_{r-1}}\right)^{L_{r-1}}<e<3 .
$$

This means by Lemma 9.4 that

$$
R_{n}\left(A_{\ell_{r}}^{L_{r-1}}\right) \leq 2 n^{1 / r}+1 .
$$

Thus

$$
\lim _{n \rightarrow \infty} \frac{\log R_{n}\left(A_{\ell_{r}}^{L_{r-1}}\right)}{\log n}=0 \quad \text { and so } \quad \rho(\Gamma)=\lim _{n \rightarrow \infty} \frac{\log R_{n}(\Gamma)}{\log n}=0
$$

as promised.

\section{Lattices in the same semisimple group}

The following theorem gives some support to our Conjecture 1.5 which predicts the same abscissa of convergence for lattices in the same semisimple locally compact group.

Let $H=\prod_{i=1}^{\ell} \operatorname{SL}_{2}\left(K_{i}\right)$ where each $K_{i}$ is a local field. Recall that rk $H=\ell$, and when $\ell \geq 2$, every irreducible lattice $\Gamma$ in $H$ is $(S$-)arithmetic. In this case, Serre's conjecture $[\mathrm{Se}]$ predicts that $\Gamma$ has the CSP. This has been proved in the case of non-uniform lattices. On the other hand, when $\ell=1$, there are non-arithmetic lattices, and even the arithmetic ones do not satisfy the CSP (see [LS. Chapter 7] for an overview and references). Here we prove

Theorem 10.1. Let $H=\prod_{i=1}^{\ell} \mathrm{SL}_{2}\left(K_{i}\right)$ where the $K_{i}$ are local fields not of characteristic 2. Let $\Gamma$ be an irreducible lattice of $H$. Then:

(a) If $\ell=1$, then $\rho(\Gamma)=\infty$.

(b) If $\ell \geq 2$ and $\Gamma$ has the CSP, then $\rho(\Gamma)=2$.

Before proving the theorem, let us make a few observations on the connection between representation growth and subgroup growth of a finitely generated pro- $p$ group $L$. As before, let $a_{n}(L)$ (resp. $\left.s_{n}(L)\right)$ be the number of subgroups of $L$ of index $n$ (resp. at most $n$ ) and $r_{n}(L)$ (resp. $R_{n}(L)$ ) the number of irreducible representations of $L$ of degree $n$ (resp. at most $n$ ). For a finite index subgroup $M$ of $L$, define

$$
\begin{aligned}
d(M) & =\operatorname{dim}_{\mathbb{F}_{p}}\left(M /[M, M] M^{p}\right)=\log _{p}\left(\left|M /[M, M] M^{p}\right|\right), \\
e(M) & =\log _{p}(|M /[M, M]|) .
\end{aligned}
$$

Let

$$
\begin{gathered}
d_{j}(L)=\sup \left\{d(M) \mid[L: M]=p^{j}\right\}, \quad e_{j}(L)=\sup \left\{e(M) \mid[L: M]=p^{j}\right\}, \\
d_{j}^{*}(L)=\sum_{i=0}^{j} d_{i}(L) .
\end{gathered}
$$


Proposition 10.2. Let $L$ be a finitely generated pro-p group and $j \in \mathbb{N}$. Then:

(a) $p^{d_{j-1}(L)-1} \leq a_{p^{j}}(L) \leq p^{d_{j-1}^{*}(L)}$.

(b) $R_{p^{j}}(L) \geq \frac{1}{p^{j}} p^{d_{j}(L)}$.

(c) $\log R_{p^{j}}(L) \geq \frac{1}{j} \log a_{p^{j}}(L)-\frac{j-1}{2}$.

(d) $r_{p^{j}}(L) \leq a_{p^{j}}(L) \cdot e_{j}(L)$.

Proof. (a) follows from [LS, Proposition 1.6.2] while (b) follows from Proposition 4.4 above. Now, by applying (a) and then (b) we have

$$
a_{p^{j}}(L) \leq \prod_{i=0}^{j-1} p^{d_{i}(L)} \leq \prod_{i=0}^{j-1} p^{i} R_{p^{i}}(L),
$$

which gives (c). Finally, (d) follows from the fact that a finite $p$-group is an $M$-group ([ᄑ]), i.e. every irreducible representation of it of degree $p^{j}$ is induced from a one-dimensional character of some subgroup of index $p^{j}$.

Corollary 10.3. If the subgroup growth rate of $L$ is faster than $n^{\log n}$ (i.e. $\left.\lim \sup \log s_{n}(L) /(\log n)^{2}=\infty\right)$ then $L$ does not have polynomial representation growth, i.e. $\rho(L)=\infty$.

The corollary follows from Proposition 10.2 (c). We should remark that this corollary is the best possible: it is shown in [LuMr] that $\mathrm{SL}_{d}\left(\mathbb{F}_{p}[[t]]\right)$ (which is a virtually pro- $p$ group) has polynomial representation growth, while its subgroup growth is $n^{\log n}$ (see [LS, Chapter 4]).

Let us now use the above observations to treat the special case of Theorem 10.1 (a) when $H=\mathrm{SL}_{2}(\mathbb{C})$ and $\Gamma$ is a cocompact lattice in $H$. A well known conjecture, attributed to Thurston, asserts that in this case, $\Gamma$ has a finite index subgroup $\Delta$ which maps onto $\mathbb{Z}$. This would give our claim immediately. However, the conjecture remains wide open. Still, it was shown in [Lu1] that such $\Gamma$ has a finite index subgroup whose pro- $p$ completion $L$ is a Golod-Shafarevich group (i.e. $d(L) \geq 4$ while $r(L)<d(L)^{2} / 4$ where $r(L)$ is the minimal number of pro- $p$ relations of $L$, i.e. $\left.r(L)=\operatorname{dim} H^{2}\left(L, \mathbb{F}_{p}\right)\right)$. For such groups, Shalev (cf. [LS. Theorem 4.6.4]) proved that for every $\epsilon>0, a_{n}(L) \geq n^{(\log n)^{2-\epsilon}}$ for infinitely many integers $n$. Thus Corollary 10.3 implies that $\rho(\Gamma) \geq \rho(L)=\infty$.

We mention in passing that Shalen and Wagreich (see [SW, Lemma 1.3]) proved a slightly better estimate on $d_{j}(\Gamma)$ (and hence on $a_{n}(\Gamma)$ ). A much better estimate was given recently by Lackenby [Ly].

Now, to complete the proof of (a) of the theorem, we recall that in all other cases, the analogue of Thurston's conjecture is true. In fact, it is even known (by several different methods of proof; see discussion in [LS, \$7.3]) that in all these cases $\Gamma$ has a finite index subgroup which is mapped onto a non-abelian free group. Thus, clearly, $\rho(\Gamma)=\infty$. 
For (b), by Corollary 4.5 and Proposition 4.6 we may assume without loss of generality that

$$
\mathcal{Z}_{\Gamma}(s)=\mathcal{Z}_{\mathbf{G}(\mathbb{C})}(s)^{\# S_{\infty}} \cdot \prod_{v \notin S} \mathcal{Z}_{L_{v}}(s)
$$

where $G(\mathbb{C})=\mathrm{SL}_{2}(\mathbb{C})$ and all but finitely many $L_{v}$ are of the form $\mathrm{SL}_{2}\left(\mathcal{O}_{v}\right)$ where $\mathcal{O}_{v}$ is the ring of integers of the completion of the global field $k$ at $v$, and the remaining $L_{v}$ are compact open subgroups of groups which are either of the form $\mathrm{SL}_{2}$ of a local field, or $\mathrm{SL}_{1}$ of a quaternion algebra over a local field. The Euler factors corresponding to these remaining factors have abscissa of convergence 1 by Theorems 6.3, 7.1 and 7.3 In determining whether $\mathcal{Z}_{\Gamma}(s)$ does or does not have abscissa of convergence 2 , they may therefore be omitted from the Euler product. Likewise, $\mathcal{Z}_{\mathbf{G}(\mathbb{C})}(s)$ has abscissa of convergence 1 by Theorem 5.1, so the first factor on the right hand side of 10.1 may be omitted from the Euler product. It remains to consider the abscissa of convergence of

$$
\prod_{v \notin T} \mathcal{Z}_{\mathrm{SL}_{2}\left(\mathcal{O}_{v}\right)}(s)
$$

for some finite set $T$ of places of $k$.

As the $K_{i}$ are not of characteristic 2, the same is true for $k$ and therefore for the $k_{v}$. For $s$ in the interval $[2,3]$, we have $2^{s} \leq 8$, and

$$
(q+1)^{-s}<q^{-s}<(q-1)^{-s} \leq 8 q^{-s} .
$$

By Theorem 6.3 , for $q$ odd,

$$
\begin{aligned}
\mathcal{Z}_{\mathrm{SL}_{2}\left(\mathcal{O}_{v}\right)}(s) & >1+\left(q^{-s}+\frac{q-1}{2}(q-1)^{-s}\right)+\frac{4 q\left(\frac{q^{2}-1}{2}\right)^{-s}+\frac{q^{2}-1}{2}\left(q^{2}-q\right)^{-s}}{1-q^{1-s}} \\
& >1+\frac{q}{2} q^{-s}+\frac{\frac{q^{2}}{2}\left(q^{2}\right)^{-s}}{1-q^{1-s}} \\
& =1+\frac{1}{2} q^{1-s}+\frac{1}{2}\left(q^{1-s}\right)^{2}\left(1-q^{1-s}\right)^{-1}>\left(1-q^{1-s}\right)^{-1 / 2}
\end{aligned}
$$

In the other direction, we have

$$
\begin{aligned}
\mathcal{Z}_{\mathrm{SL}_{2}\left(\mathcal{O}_{v}\right)}(s)< & 1+q^{-s}+q^{1-s}+16 q^{-s}+4 q^{1-s}+128 q^{-s} \\
& +\frac{256 q^{1-2 s}+4 q^{2-2 s}+q^{2-2 s}}{1-q^{1-s}} \\
< & 1+100 q^{1-s}+\frac{1000 q^{2-2 s}}{1-q^{1-s}}<\left(1-q^{1-s}\right)^{-100}
\end{aligned}
$$

There are finitely many Euler factors for which $q$ is even (and none at all if $k$ is of positive characteristic). We may therefore assume $q$ is odd for all Euler factors and prove that $\mathcal{Z}_{\Gamma}(s)$ converges for $s>2$ and diverges for $s=2$ by comparing the product $(10.2)$ with $\zeta_{k, T}(s-1)^{1 / 2}$ and $\zeta_{k, T}(s-1)^{100}$, where $\zeta_{k, T}(s)$ is the usual Dedekind $\zeta$-function of $k$ with the Euler factors at $T$ removed (which is analytic for $\Re(s)>1$ and has a simple pole at $s=1)$. 


\section{Remarks and suggestions for further research}

Clearly, we are still at the qualitative stage in our understanding of the abscissa of convergence for representation zeta functions. We mention some of the questions left open by this paper.

For general finitely generated groups $\Gamma$, are there any positive values which cannot be achieved? For infinite linear groups, $1 / 15$ is probably not optimal. A better understanding of $\rho(U)$ where $U$ is a compact open subgroup of $E_{8}\left(k_{v}\right)$ seems likely to improve that value. We do not even have a conjecture regarding the greatest lower bound.

For arithmetic groups $\Gamma$ satisfying the congruence subgroup property, we still lack a plausible conjecture for the value of $\rho(\Gamma)$. It is conceivable that without determining the actual value, one can prove that $\rho(\Gamma)$ is always rational in this setting. We do not know if the values $\rho(\Gamma)$ as $\Gamma$ ranges over arithmetic groups satisfying the CSP are bounded above. By combining the results of [LiSh2] with upper bound estimates of the kind developed in Theorem 7.3 it is likely that one can prove

$$
\rho(\Gamma) \leq c+\sup _{v} \rho\left(\Gamma_{v}\right)
$$

where $\Gamma_{v}$ denotes the $v$-adic completion of $\Gamma$ and $c$ is an absolute constant.

This raises the question as to whether one can find reasonable upper bounds for $\rho(U)$ for compact open subgroups $U \subset \mathbf{G}(K)$ of almost simple algebraic groups over nonarchimedean local fields. For instance, is there an absolute constant which works for all $\mathbf{G}$ and all $K$ ? In a different direction, can one prove equality for the values of $\rho$ for groups of fixed type ( $\mathrm{SL}_{n}$ for example), as $K$ ranges over local fields? (Compare Theorem 6.3 . Theorem 7.1, and [LuNi].) It is conceivable that one could do so without being able to compute the common value. As a step toward computing $\rho\left(\mathrm{SL}_{n}\left(\mathbb{Z}_{p}\right)\right)$, it would be interesting to estimate the number of conjugacy classes in $\mathrm{SL}_{n}\left(\mathbb{Z} / p^{r} \mathbb{Z}\right)$, for instance when $n$ and $p$ are fixed and $r$ is allowed to grow.

One approach to these problems would be to try to imitate the method of Theorem 7.1 Let $\mathbf{G}$ be a group scheme of finite type over the ring $\mathcal{O}_{K}$ of integers in a local field $K$ with almost simple generic fiber. Let $U=\mathbf{G}\left(\mathcal{O}_{K}\right)$ and let $U_{r}$ denote the kernel of $U \rightarrow \mathbf{G}\left(\mathcal{O}_{K} / \pi_{K}^{r}\right)$. Every element of $U / U_{r}$ lifts to a regular semisimple element of $U$. Up to $\mathbf{G}(K)$-conjugacy, there are finitely many maximal tori $\mathbf{T}_{i}$ in the generic fiber of $\mathbf{G}$, and any regular semisimple conjugacy class meets exactly one such maximal torus, and meets it in finitely many points. The conjugacy classes of $U$ up to $\mathbf{G}(K)$-conjugacy are what gives rise to the general lower bound of Proposition 6.6 .

Describing the regular semisimple conjugacy classes in $U$ (rather than $\mathbf{G}(K)$ ) brings the Bruhat-Tits building $\mathcal{B}$ of $\mathbf{G}$ over $K$ into the picture. (Note that for anisotropic groups, where the building is trivial, Theorem 7.1 says that Proposition 6.6 is sharp.) For simplicity, let us suppose that $U$ is exactly the stabilizer of a vertex $x_{0}$ of the building. If, for example, $g \in U_{r}$, then it fixes all the vertices in $B_{x_{0}}(r)$, the ball of radius $r$ centered at $x_{0}$ in $\mathcal{B}$. Now, if $h_{i} \in \mathbf{G}(K), i=1,2$, and $h_{i}\left(x_{0}\right) \in B_{x_{0}}(r)$, then $h_{i}^{-1} g h_{i}$ fixes $x_{0}$ and therefore lies in $U$. But $h_{1}^{-1} g h_{1}$ and $h_{2}^{-1} g h_{2}$ are not necessarily conjugate to each other in $U$. If $g$ is regular semisimple, then

$$
u^{-1}\left(h_{1}^{-1} g h_{1}\right) u=h_{2}^{-1} g h_{2}
$$


is equivalent to $h_{2} u^{-1} h_{1}^{-1} \in Z_{\mathbf{G}(K)}(g)=\mathbf{T}(K)$, where $\mathbf{T}$ is the unique maximal torus containing $g$. In other words, $h_{2}$ belongs to the double coset $\mathbf{T}(K) h_{1} U$, or, yet again, $h_{2}\left(x_{0}\right)$ lies in the $\mathbf{T}(K)$-orbit of $h_{1}\left(x_{0}\right)$. Thus, counting torus orbits in balls in the building is closely connected with the problem of classifying conjugacy classes in $U$ and thereby with the problem of counting conjugacy classes in $U / U_{r}$.

This strongly suggests that when the building $\mathcal{B}$ is "larger", there are more conjugacy classes in $U$ (and $U / U_{r}$ ) and $\rho(U)$ tends to be larger. As mentioned above, it is still not clear if $\rho(U)$ can be arbitrarily large. A good test case: is $\rho\left(\mathrm{SL}_{n}\left(\mathbb{Z}_{p}\right)\right)$ bounded above independently of $n$ ?

Added in proof. Kassabov and Nikolov [KN] have improved on Theorem 9.2, giving a positive answer to the question in $\S 11$ as to whether every real number is the abscissa of convergence of the representation zeta function of some finitely generated group.

\section{References}

[BLMM] Bass, H., Lubotzky, A., Magid, A., Mozes, S.: The proalgebraic completion of rigid groups. In: Proc. Conf. Geometric and Combinatorial Group Theory, Part II (Haifa, 2000), Geom. Dedicata 95, 19-58 (2002) Zbl 1059.20036 MR 1950883

[BMS] Bass, H., Milnor, J., Serre, J.-P.: Solution of the congruence subgroup problem for $\mathrm{SL}_{n}(n \geq 3)$ and $\mathrm{Sp}_{2 n}(n \geq 2)$. Inst. Hautes Études Sci. Publ. Math. 33, 59-137 (1967) Zbl 0174.05203 MR 0244257

[BLR] Bosch, S., Lütkebohmert, W., Raynaud, M.: Néron Models. Ergeb. Math. Grenzgeb. (3) 21, Springer, Berlin (1990) Zbl 0705.14001 MR 1045822

[Cd] Corduneanu, C.: Almost Periodic Functions. Tracts in Pure Appl. Math. 22, Interscience, New York (1968) Zbl 0175.09101 MR 0481915

[Co] Corlette, K.: Archimedean superrigidity and hyperbolic geometry. Ann. of Math. 135, 165-182 (1992) Zbl 0768.53025 MR 1147961

[De] Denef, J.: The rationality of the Poincare series associated to the $p$-adic points on a variety. Invent. Math. 77, 1-23 (1984) Zbl 0537.12011 MR 0751129

[DDMS] Dixon, J. D., du Sautoy, M. P. F., Mann, A., Segal, D.: Analytic Pro- $p$-Groups. London Math. Soc. Lecture Note Ser. 157, Cambridge Univ. Press, Cambridge (1991) Zbl 0744.20002 MR 1152800

[DG] du Sautoy, M., Grunewald, F.: Zeta functions of groups and rings. In: Proc. ICM, Madrid 2006, Vol. II, 131-149 Zbl 1112.20024 MR 2275592

[GS] Gromov, M., Schoen, R.: Harmonic maps into singular spaces and $p$-adic superrigidity for lattices in groups of rank one. Inst. Hautes Études Sci. Publ. Math. 76, 165-246 (1992) Zbl 0896.58024 MR 1215595

[HM] Hochschild, G., Mostow, G. D.: Representations and representative functions of Lie groups. Ann. of Math. 66, 495-542 (1957) Zbl 0080.25101 MR 0098796

[Ho] Howe, R. E.: Kirillov theory for compact $p$-adic groups. Pacific J. Math. 73, 365-381 (1977) Zbl 0385.22007 MR 0579176

[Hu] Humphreys, J. E.: Introduction to Lie Algebras and Representation Theory. Springer, New York (1978) Zbl 0447.17001 MR 0499562

[I] Isaacs, M. I.: Character Theory of Finite Groups. Dover Publ., New York (1994). Zbl 0849.20004 MR 1280461 
[Ja1] Jaikin-Zapirain, A.: On the number of conjugacy classes in finite $p$-groups. J. London Math. Soc. 68, 699-711 (2003) Zbl 1062.20020 MR 2009445

[Ja2] Jaikin-Zapirain, A.: Zeta function of representations of compact $p$-adic analytic groups. J. Amer. Math. Soc. 19, 91-118 (2006) Zbl 1092.20023 MR 2169043

[KN] Kassabov, M., Nikolov, N.: Cartesian products as profinite completions. Int. Math. Res. Not. 2006, Art. ID 72947, 17 pp. Zbl 1129.20019 MR 2264720

[Ly] Lackenby, M.: New lower bounds on subgroup growth and homology growth. arXiv: math.GR/0512261

[La] Larsen, M.: Determining a semisimple group from its representation degrees. Int. Math. Res. Not. 2004, no. 38, 1989-2016 Zbl 1073.22009 MR 2063567

[LaLu] Larsen, M., Lubotzky, A.: Normal subgroup growth of linear groups: the $\left(G_{2}, F_{4}, E_{8}\right)$ theorem. In: Algebraic Groups and Arithmetic, Tata Inst. Fund. Res., Mumbai, 441-468 (2004) MR 2094120

[LiSh1] Liebeck, M. W., Shalev, A.: Fuchsian groups, coverings of Riemann surfaces, subgroup growth, random quotients and random walks. J. Algebra 276, 552-601 (2004) Zbl 1068.20052 MR 2058457

[LiSh2] Liebeck, M. W., Shalev, A.: Character degrees and random walks in finite groups of Lie type. Proc. London Math. Soc. 90, 61-86 (2005) Zbl 1077.20020 MR 2107038

[Lu1] Lubotzky, A.: Group presentation, $p$-adic analytic groups and lattices in $\mathrm{SL}_{2}(\mathbb{C})$, Ann. of Math. 118, 115-130 (1983) Zbl 0541.20020 MR 0707163

[Lu2] Lubotzky, A.: Lattices in rank one Lie groups over local fields. Geom. Funct. Anal. 1, 406-431 (1991) Zbl 0786.22017 MR 1132296

[LuMg] Lubotzky, A., Magid, A. R.: Varieties of representations of finitely generated groups. Mem. Amer. Math. Soc. 58, no. 336 (1985) Zbl 0598.14042 MR 0818915

[LuMr] Lubotzky, A., Martin, B.: Polynomial representation growth and the congruence subgroup problem. Israel J. Math. 144, 293-316 (2004) Zbl pre02147131 MR 2121543

[LuNi] Lubotzky, A., Nikolov, N.: Subgroup growth of lattices in semisimple Lie groups. Acta Math. 193, 105-139 (2004) Zbl 02183715 MR 2121543

[LS] Lubotzky, A., Segal, D.: Subgroup Growth. Progr. Math. 212, Birkhäuser, Basel (2003) Zbl 1071.20033 MR 1978431

[Ma] Margulis, G. A.: Discrete Subgroups of Semisimple Lie Groups. Ergeb. Math. Grenzgeb. (3) 17, Springer, Berlin (1991) Zbl 0732.22008 MR 1090825

[Pi] Pink, R.: Compact subgroups of linear algebraic groups. J. Algebra 206, 438-504 (1998) Zbl 0914.20044 MR 1637068

[PR] Platonov, V., Rapinchuk, A.: Algebraic Groups and Number Theory. Pure Appl. Math. 139, Academic Press, Boston, MA (1994) Zbl 0841.20046 MR 1278263

[Pr] Prasad, G.: Strong approximation for semi-simple groups over function fields. Ann. of Math. 105, 553-572 (1977) Zbl 0348.22006 MR 0444571

[Ra] Raghunathan, M. S.: On the congruence subgroup problem. Inst. Hautes Études Sci. Publ. Math. 46, 107-161 (1976) Zbl 0347.20027 MR 0507030

[Ri] Ribes, L.: Introduction to Profinite Groups and Galois Cohomology. Queen's Papers in Pure Appl. Math. 24, Queen's Univ., Kingston, Ont. (1970) Zbl 0948.11043 MR 0260875

[S] Segal, D.: The finite images of finitely generated groups. Proc. London Math. Soc. 82, 597-613 (2001) Zbl 1022.20011 MR 1816690

[Se] Serre, J.-P.: Le problème des groupes de congruence pour $\mathbf{S L}_{2}$. Ann. of Math. 92, 489527 (1970) Zbl 0239.20063 MR 0272790 
[SW] Shalen, P. B., Wagreich, P.: Growth rates, $\mathbb{Z}_{p}$-homology and volumes of hyperbolic 3-manifolds. Trans Amer. Math. Soc. 331, 895-917 (1992) Zbl 0768.57001 MR 1156298

[Ti] Tits, J.: Classification of algebraic semisimple groups. In: Algebraic Groups and Discontinuous Subgroups (Boulder, CO, 1965), Proc. Sympos. Pure Math., Amer. Math. Soc., Providence, RI, 33-62 (1966) Zbl 0238.20052 MR 0224710

[Wi] Witten, E.: On quantum gauge theories in two dimensions. Comm. Math. Phys. 141, 153-209 (1991) Zbl 0762.53063 MR 1133264 